\title{
Stokes diagnostics of simulations of magnetoconvection of mixed-polarity quiet-Sun regions
}

\author{
E. V. Khomenko ${ }^{1,2}$, S. Shelyag ${ }^{3}$, S. K. Solanki ${ }^{3}$, and A. Vögler ${ }^{3}$ \\ 1 Instituto de Astrofísica de Canarias, 38205, C/ Vía Láctea s/n, Tenerife, Spain \\ 2 Main Astronomical Observatory, NAS, 03680 Kyiv, Zabolotnogo str. 27, Ukraine \\ e-mail: khomenko@iac.es \\ 3 Max-Planck-Institut für Sonnensystemforschung, 37191 Katlenburg-Lindau, Germany, \\ e-mail: shelyag@mps.mpg.de, solanki@mps .mpg .de , avoegler@mps .mpg . de
}

Received 1 March 2005 / Accepted 11 May 2005

\section{ABSTRACT}

Realistic solar magneto-convection simulations including the photospheric layers are used to study the polarization of the Fe I Zeeman-sensitive spectral lines at $6301.5,6302.5,15648$ and $15652 \AA$. The Stokes spectra are synthesized in a series of snapshots with a mixed-polarity magnetic field whose average unsigned strength varies from $\langle B\rangle=10$ to $140 \mathrm{G}$. The effects of spatial resolution and of the amount of magnetic flux in the simulation box on the profiles shapes, amplitudes and shifts are discussed. The synthetic spectra show many properties in common with those observed in quiet solar regions. In particular, the simulations reproduce the width and depth of spatially averaged Stokes $I$ profiles, the basic classes of the Stokes $V$ profiles and their amplitude and area asymmetries, as well as the abundance of the irregular-shaped Stokes $V$ profiles. It is demonstrated that the amplitudes of the $1.56 \mu \mathrm{m}$ lines observed in the inter-network are consistent with a "true" average unsigned magnetic field strength of $20 \mathrm{G}$. We show that observations using these and visible lines, carried out under different seeing conditions (e.g., simultaneous observations at different telescopes), may result in different asymmetries and even opposite polarities of the profiles in the two spectral regions observed at the same spatial point.

Key words. magnetohydrodynamics (MHD) - Sun: magnetic fields - Sun: infrared - polarization - Sun: photosphere

\section{Introduction}

One widely used approach for studying the magnetic field on the Sun is based on the interpretation of observations of Stokes spectra (for a review see Solanki 1993). Such spectra contain the most detailed information on the structure and dynamics of magnetized photospheric plasma and its interaction with convection, i.e. magnetoconvection. Stokes profiles observed in the quiet Sun have a broad range of asymmetries and show a variety of shapes (Sigwarth et al. 1999; Sánchez Almeida \& Lites 2000; Khomenko et al. 2003). The observations of mainly network fields in the Fe I 6301 and $6302 \AA$ lines show that the average amplitude asymmetry, $\delta a$, and area asymmetry, $\delta A$, of Stokes $V$ profiles are about $15 \%$ and $5 \%$ (Grossmann-Doerth et al. 1996; Sigwarth et al. 1999). The Stokes $V$ asymmetries of the inter-network fields determined from infrared $\mathrm{Fe}$ I lines at $1.56 \mu \mathrm{m}$ are similar $(\overline{\delta a}=15 \%$ and $\overline{\delta A}=7 \%$, see Khomenko et al. 2003). Nearly $30 \%$ of all the significant $V$ profiles of the infrared lines and $35 \%$ of the visible lines are "anomalous" (i.e. without the usual double-lobe shape) suggesting the existence of mixed magnetic polarities within one resolution element (Socas-Navarro \& Sánchez Almeida 2002; Khomenko et al. 2003). The fraction of "anomalous" profiles is known to increase with decreasing magnetic flux. There are indications of the dependence of the asymmetries on LOS velocities and continuum intensities (Sigwarth et al. 1999; Khomenko et al. 2003; Socas-Navarro et al. 2004). The distribution of the velocities determined from Stokes $V$ zero-crossing, $V_{z c}$, of the inter-network fields shows a large scatter from -5 to $+5 \mathrm{~km} \mathrm{~s}^{-1}$ with almost no average redshift (Khomenko et al. 2003) while the mainly network data of Grossmann-Doerth et al. (1996) and Sigwarth et al. (1999) give $\overline{V_{z c}}$ of 970 and $730 \mathrm{~m} \mathrm{~s}^{-1}$, respectively.

As was first noted by Illing et al. (1975), Stokes profiles are asymmetric if velocity and magnetic field gradients along the line of sight are present. Since then, various models which involve dynamic processes inside and outside the magnetic elements and specific configurations of the magnetic field accompanied by mass flows have been proposed (see Sigwarth et al. 1999, for an overview). The concept of a vertical flux tube, with magnetic field lines fanning out with height, that is surrounded by a convective downflow was exploited by Grossmann-Doerth et al. (1988) and Solanki (1989), who showed that the Stokes $V$ profiles of such a configuration are asymmetric without significant wavelength shifts. Allowing for strong downflows with gradients inside a flux tube permitted Bellot Rubio et al. (1997) to fit the asymmetries of ASP Stokes profiles observed in a plage region. The amplitude asymmetries appeared to be 
significantly larger than the area asymmetries, in agreement with observations of Stokes $V$ at the disc centre. Alternatively, Frutiger \& Solanki (1998) showed that the observed asymmetry can also be reproduced by the overlap of different flows within and outside the flux tube, with no net mean flow within the flux tube. The basic principles of the formation of asymmetric Stokes $V$ profiles in the presence of a magnetopause, including a possible formation mechanism of extreme asymmetries, were described by Steiner (2000). The MIcro Structured Magnetic Atmosphere (MISMA) concept was put forward by Sánchez Almeida et al. (1996), who argued that this model is able to account for the observed range of asymmetries in the quiet Sun irrespective of the abnormality of the profiles.

The approach described above is based on the a priori assumption of the magnetic field structure. The information is inferred from spatially unresolved profiles within the framework of a model whose basic structure is prescribed. Thus, a clear shortcoming of this approach is that the results can be biased according to the adopted model concept.

Another approach for studying magnetic fields in the Sun is based on the numerical modeling of magnetoconvection (see the reviews by Schüssler 2001, 2003, and references therein). Realistic magneto-convection simulations involve the solution of the full compressible MHD equations including elaborate physics, such as multidimensional radiative transfer or partial ionization and, thus, can make clear predictions about the complex processes that take place in the Sun's magnetized atmosphere. The comparison of the results of simulations with observations allows conclusions to be drawn about their realism, but may also provide guidance on the interpretation of observations. In order to compare the models with observations, spectral Stokes diagnostics are required. One of the most sensitive parameters of Stokes profiles is the Stokes $V$ asymmetry. As pointed out above, the observed Stokes $V$ profiles exhibit a very broad range of asymmetry. Although a systematic statistical study has not been performed yet, many types of observed strongly asymmetric profiles can also be found in simulations. Sigwarth et al. (1999) state that the asymmetries observed by them in a quiet solar area are in quantitative agreement with the results from the 2D numerical simulations of Steiner et al. (1998); Steiner (1999) and Grossmann-Doerth et al. (1998). Since 2D and 3D simulations can differ significantly it is important to test this also with $3 \mathrm{D}$ simulations. Recently, Sánchez Almeida et al. (2003a) claimed that the velocity and magnetic field gradients in 3D MHD simulations are too small compared to the real Sun. They used idealized MHD turbulent dynamo simulations by Cattaneo (1999); Emonet \& Cattaneo (2001). Since these numerical simulations are not specially designed for spectral synthesis, the authors had to apply an arbitrary scaling to the velocity and the magnetic field from the simulations and assume a Milne-Eddington atmosphere for the thermodynamic variables. These ad hoc components of the computations of Sánchez Almeida et al. (2003a) correspond to a rather severe departure from a self consistent treatment. An investigation of realistic radiation MHD simulations is needed in order to clarify whether the claim of Sánchez Almeida et al. (2003a) has any basis, but also in order to classify the nature of quiet Sun magnetic fields.
In the present paper we employ the most recent realistic 3D simulations of magnetoconvection (Schüssler 2003; Vögler et al. 2005) and synthesize the Stokes spectra of the following photospheric lines Fe I 6301.5, 6302.5, 15648 and $15652 \AA$ for selected snapshots. The amplitudes, asymmetries, shapes and shifts of the polarized spectra are studied as a function of the amount of magnetic flux in the MHD model box. We also study the influence of artificially degrading the spatial resolution. Since we are interested in the properties of the quiet Sun, we employ a simulation which studies the development of a bipolar magnetic field distribution.

The paper is organized as follows. Sections 2 and 3 describe the simulations and spectral synthesis. The shape of Stokes $V$ profiles and the number of irregularly-shaped profiles are analyzed with the help of the Principal Component Analysis in Sect. 4. The amplitudes of the polarization signals are compared with observations in Sect. 5. The asymmetries and zero-crossing shifts are discussed in Sects. 6 and 7. We use the simulations to predict the results that one would expect from the simultaneous observations of visible and IR lines under different seeing conditions in Sect. 8. The main results are summarized in Sect. 9.

\section{Radiative MHD simulations}

"Realistic" solar magneto-convection simulations aim at representing radiative and magnetohydrodynamical processes in the solar photosphere and the uppermost layers of the convection zone with sufficient accuracy that the results can be directly compared with the observations. We have used the MURAM ${ }^{1}$ code, a 3D MHD code which includes non-grey radiative transfer, full compressibility, and the effects of partial ionization for the 11 most abundant chemical elements (Vögler et al. 2003, 2005).

The size of the computational domain for the simulations considered here is $6000 \times 6000 \times 1400 \mathrm{~km}^{3}$ which is covered by $288 \times 288 \times 100$ grid points. The domain has periodic side boundaries, a closed top and an open bottom boundary (for details see Vögler 2003). The simulation started with a planeparallel solar model atmosphere (Spruit 1974), extending between $800 \mathrm{~km}$ below and $600 \mathrm{~km}$ above the level of optical depth $\tau=1$ at $500 \mathrm{~nm}$, as initial condition. Then purely hydrodynamical convection $(B=0)$ was allowed to develop. After it reached a statistically steady state, a homogeneous vertical magnetic field was introduced (at about $60 \mathrm{~min}$ after the start of the simulation).

We concentrate here on a simulation run that has a bi-polar structure of the magnetic field. It aims at simulating the bipolar magnetic field in quiet solar regions. In this run, the computation box was split into 4 parts. The initial magnetic field had opposite polarity in adjacent parts. Initially, the unsigned magnetic field strength was 200 G. Within a few minutes of

1 The MURAM (MPS/University of Chicago RAdiative MHD) code has been developed by the MHD simulation groups at the Max-Planck-Institut für Sonnensystemforshung Katlenburg-Lindau (A. Vögler, S. Shelyag, M. Schüssler) and at the University of Chicago (F. Cattaneo, Th. Emonet, T. Linde). 

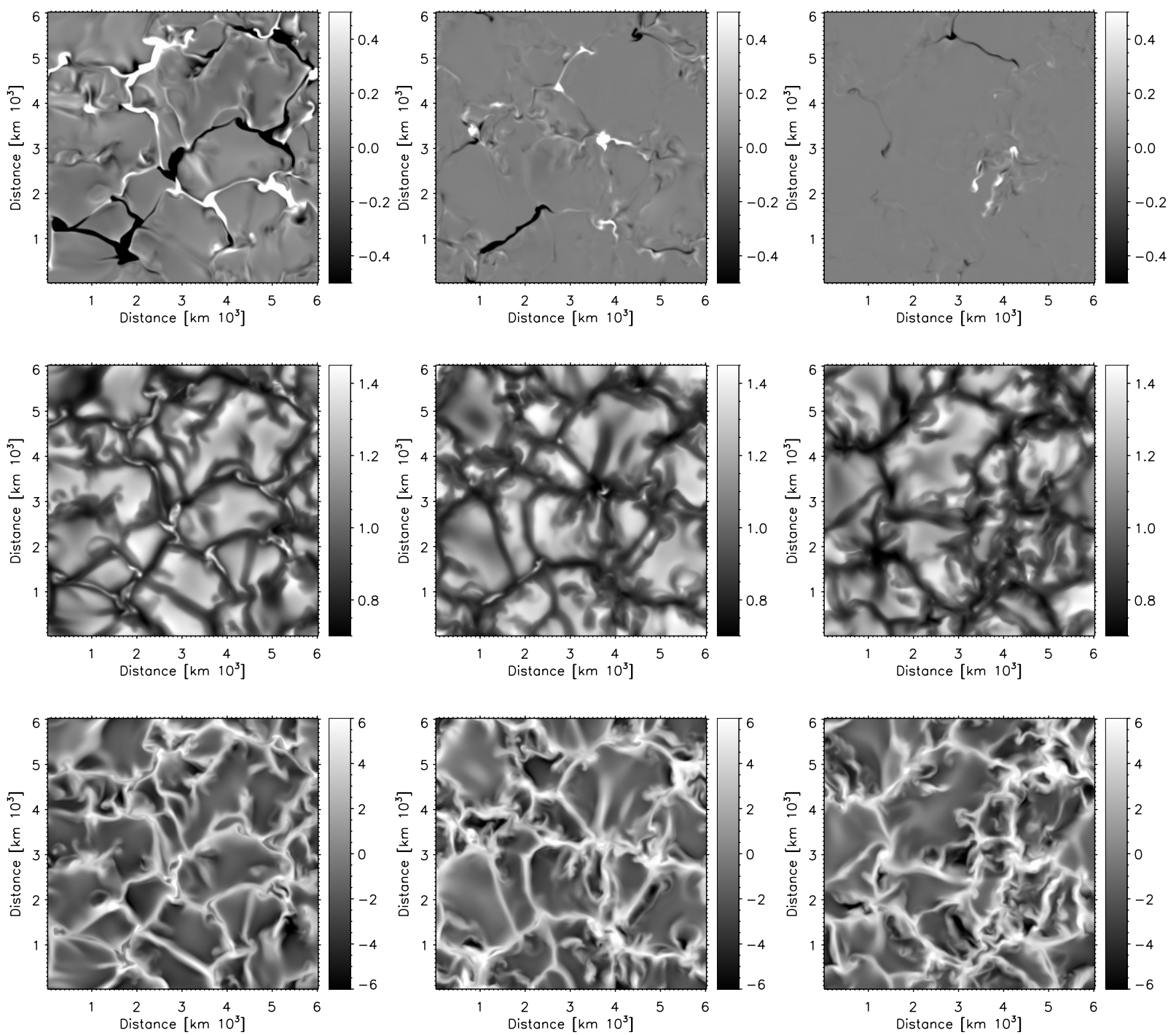

Fig. 1. Maps of physical quantities of the snapshots with $\langle B\rangle=140 \mathrm{G}$ (left panels), $30 \mathrm{G}$ (middle panels) and $10 \mathrm{G}$ (right panels). Upper panels: vertical component of the magnetic field at the level $\log \tau_{5000}=0$, corresponding to the visible solar surface. Middle panels: normalized continuum intensity $\delta I_{\mathrm{c}}=\left(I_{\mathrm{c}}-\bar{I}_{\mathrm{c}}\right) / \bar{I}_{\mathrm{c}}$ at $630 \mathrm{~nm}$. The first two images show local brightenings in the magnetic flux concentrations in intergranular lanes. Lower panels: vertical velocity component, positive values are downflows.

simulated time, most of the magnetic flux assembled in the downflow regions of the convection pattern. The redistribution of the existing magnetic field by convective motions led to the cancellation of elements with different polarities. This produced an almost exponential decrease with time of the average unsigned magnetic field in the computational box. The snapshots used in the present work were taken 17, 36, 112, 169 and $237 \mathrm{~min}$ after the magnetic field was introduced. At these moments, the average unsigned magnetic field strength in the box was 140, 80, 30, 20 and $10 \mathrm{G}$, respectively (at $\log \tau=-1$ ). The first two snapshots could represent bi-polar solar network or enhanced network regions, while the latter three snapshots with the lower field strength should better correspond to internetwork regions. Below we will use the notation $\langle B\rangle$ for the spatially averaged unsigned magnetic field strength, and $\left\langle\left|B_{z}\right|\right\rangle$ for the average longitudinal magnetic field component. The latter value is lower, being $108,58,21,11$ and $6 \mathrm{G}$ at $\log \tau=-1$ for the considered snapshots.
Some quantities from the simulation snapshots are shown in Fig. 1. The magnetic field maps (upper panels of Fig. 1) show the concentrations of magnetic flux ( $B$ ranges up to $2700 \mathrm{G}$ ), which are mainly located in intergranular lanes. Note that only few intergranules possess strong magnetic field in the $10 \mathrm{G}$ snapshot. The typical spatial scale of magnetic field concentrations in this case is similar to a mesogranular scale. In the case of the snapshot with $\langle B\rangle=140 \mathrm{G}$, most magnetic flux concentrations in the intergranular lanes correspond to local continuum brightenings which are caused by the partial evacuation of magnetic flux tubes and the radiative heating of flux tube interiors by the hot neighboring granules (see Vögler \& Schüssler 2003, and references therein). These bright points are a well known feature of solar network and plage regions. Note that some bright features are also present in intergranular lanes in the much lower flux case of $\langle B\rangle=30 \mathrm{G}$. Interestingly, the existence of such bright points in a low-flux solar region was recently revealed in the high-resolution observations taken 
Table 1. Atomic parameters of the Fe I lines.

\begin{tabular}{cccc}
\hline \hline$\lambda[\AA]$ & EPL $[\mathrm{eV}]$ & $\log g f$ & $g_{\text {eff }}$ \\
\hline 6301.5012 & 3.654 & -0.718 & 1.67 \\
6302.4936 & 3.686 & -1.235 & 2.5 \\
15648.5088 & 5.426 & -0.652 & 3.0 \\
15652.8809 & 6.246 & -0.050 & 1.53 \\
\hline
\end{tabular}

inside a supergranular cell in $G$-band (see Sánchez Almeida et al. 2004). The granule structure seen in continuum brightness and vertical velocity becomes increasingly complex with decreasing magnetic flux. Note the reduction of velocities in the interior of the large-flux concentrations inside intergranular lanes in the $140 \mathrm{G}$ snapshot (bottom left panel).

\section{Spectral synthesis}

We consider Stokes profiles of the Fe I 6301.5, 6302.5, 15648 and $15652 \AA$ Zeeman-sensitive spectral lines formed at solar disc centre $(\mu=1)$. These spectral lines are extensively used for the diagnostics of solar magnetic fields in quiet and active regions (see e.g. Rabin 1992; Rüedi et al. 1992; Keller et al. 1994; Lin 1995; Grossmann-Doerth et al. 1996; Sigwarth et al. 1999; Sánchez Almeida \& Lites 2000; Khomenko et al. 2003, and references therein).

The solution of the set of radiative transfer equations for polarized light is implemented into the code STOPRO (see Solanki 1987; Solanki et al. 1992; Frutiger et al. 2000) by the method given by Rees et al. (1989). The Stokes profiles were calculated for every vertical column of the selected snapshots, corresponding to the line-of-sight direction. Prior to the calculations, in order to improve the vertical sampling, the simulations were interpolated to a finer depth scale with 87 points between the optical depths $\log \tau_{5000}=-4$ and $\log \tau_{5000}=1$. The assumption of local thermodynamic equilibrium (LTE) was made throughout in the present study. The Fe abundance used in the line calculations was 7.50. The atomic parameters of the lines are summarized in Table 1.

The laboratory wavelengths of the lines, $\lambda$, and their excitation potentials, EPL, were taken from Nave et al. (1994). The values of the oscillator strengths, $\log g f$, for the infrared and visible lines are given in the VALD database (Kupka et al. 1999; Ryabchikova et al. 1999; Piskunov et al. 1995; Kurucz 1994). Given the electronic configuration, the collisional broadening by neutral atoms is calculated using the formulation given by Anstee \& O'Mara (1995) and Barklem et al. (1998, 2000). In Table $1, g_{\text {eff }}$ is the effective Landé factor.

Figure 2 shows synthetic and observed Stokes $I$ profiles of the Fe I 6301.5, 6302.5, 15648 and $15652 \AA$ lines. The observed profiles were taken from the Liège atlas (Delbouille et al. 1973). The synthetic profiles that appear in the figure were produced by averaging over all the Stokes $I$ profiles in the considered series of snapshots. This mixture roughly mirrors the mixture of network and internetwork in the quiet Sun. The synthetic lines were shifted to the red in wavelength in order to have the same wavelength as in the atlas. This shift was of the order of $10 \mathrm{~m} \AA$ for the visible lines and $20 \mathrm{~m} \AA$ for the IR lines $\left(\sim 400-500 \mathrm{~m} \mathrm{~s}^{-1}\right)$. However, the wavelength
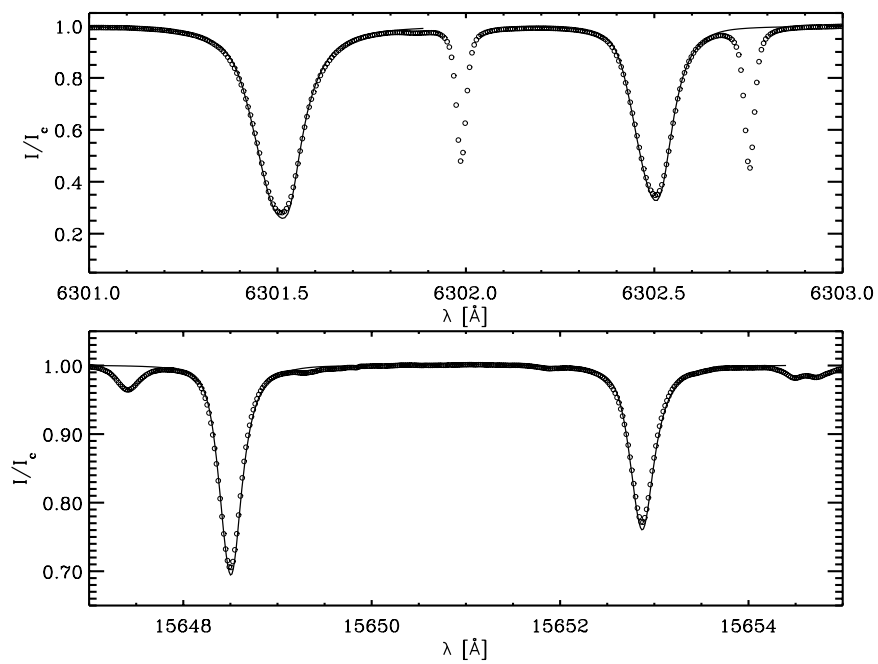

Fig. 2. Comparison between the averaged synthetic Stokes $I$ profiles (solid line) and corresponding observed ones (circles) from the Liège atlas. Upper panel: Fe I 6301 and 6302 A lines. Lower panel: Fe I 15648 and 15652 Å lines.

positions of the spectral lines in the Liège atlas contain systematic errors (Allende Prieto \& García López 1998), so not much significance should be paid to the exact value of the wavelength shift between the synthetic profiles and the atlas (see Sect. 7).

The comparison reveals that the line widths and depths are, in general, reproduced reasonably well by the simulations (note, that neither macro- no microturbulent velocity was used for the synthesis). This is an important constraint that verifies that the convective velocity field and temperature are sufficiently realistic. Note, that a good agreement between the observed and the synthetic Fe I line profiles has also been obtained with purely hydrodynamical 3D simulations (see Asplund et al. 2000; Shchukina \& Trujillo Bueno 2001). The magnetic flux at the level present in the simulations does not seem to affect to a significant extent the average Stokes $I$ profile. There is no systematic difference between the average Stokes $I$ from the snapshots with different flux levels. The existing difference seems to be oscillatory in nature.

There is some disagreement in the line depth of the synthetic lines, which turn out to be slightly deeper that the observed ones. The difference in the line depth is about $2 \%$ for the IR lines and does not exceed $10 \%$ for the visible lines. On the one hand, such a disagreement may be due to insufficient temporal-spatial statistics, since the number of granules in the computed domain is relatively small and large fluctuations may by possible. On the other hand, it could be a consequence of the closed upper boundary of the simulation box located at $600 \mathrm{~km}$. The effect of the 5-min oscillations is likely to be compensated by averaging the snapshots separated in time. Another reason for the disagreement could be an assumption of LTE that can affect the depth of the considered spectral lines (Shchukina \& Trujillo Bueno 2001). The MHD models appear to be, on average, about 100-200 K cooler in the upper layers $\left(\log \tau_{5000}>-1\right)$ as comparing to the HOLMUL solar model atmosphere (Holweger \& Müller 1974). The temperature structure of the HOLMUL semiempirical model atmosphere 
corresponds to the excitation temperature of the spectral lines (see Shchukina \& Trujillo Bueno 2001). The spectral lines synthesized under LTE in this model atmosphere have the observed solar depths, while a lower temperature will make the lines deeper as we observe.

\subsection{Spatial resolution of the numerical data}

A key objective of the present study is the statistical comparison of parameters of calculated Stokes profiles with observations. The original spatial resolution of the numerical data, i.e. the grid resolution, is equal to $20 \mathrm{~km}$, which is much better than any observations available to date. An observed signal is affected by the finite spatial resolution of the telescope and the effects of smearing caused by the turbulence of the Earth's atmosphere (seeing). Mathematically, this effect is expressed by the convolution of the solar signal by the pointspread functions (PSF) of the telescope and the atmosphere. In order to mimic this effect we convolve the computed Stokes profiles as follows. The 2D maps of emergent Stokes $I, Q, U$ and $V$ formed at each wavelength are individually convolved with the following PSF (Nordlund 1984; Schüssler et al. 2003):

$P S F=A_{\lambda, D}(r)+\frac{b}{\left(c^{2}+r^{2}\right)^{3 / 2}}$.

The first term is an Airy function describing the influence of the telescope. The FWHM (full width at half-maximum) of the Airy function depends on the telescope's diameter and the wavelength of observations, as $F W H M \simeq \lambda / D$ and, thus, different values were taken for the smoothing of the visible and infrared lines. The second part of the PSF has extended wings, so that it degrades the contrast without significantly affecting the spatial resolution of the image. By adjusting the two free parameters $b$ and $c$ we were able to make the contrast and the resolution of the smoothed image match the typical observed values, whereby the same values of the free parameters $D, b$ and $c$ are employed for all wavelengths and Stokes parameters.

\subsection{Definitions of line parameters}

We use the following notations for the line parameters under study. We use $a$ for the Stokes $V$ amplitude measured as the maximum absolute value of the circularly polarized line profile. The total area of a lobe of Stokes $V$ is denoted as $A$. The amplitude asymmetry is defined as usual, i.e. $\delta a=\left(a_{b}-a_{r}\right) /\left(a_{b}+a_{r}\right)$, where subscript " $b$ " and " $r$ " denote the blue and red wing, respectively. The same definition applies for the area asymmetry $\delta A$. The asymmetries are calculated only when this definition can be applied to a profile, i.e. when it is of regular shape (has two lobes). The wavelength shift of Stokes $I, \lambda_{I}$, is measured by parabolic fit to the line core. The zero-crossing wavelength shift of Stokes $V, \lambda_{V}$, is determined by locating a zero intensity position between the two extrema of a $V$ profile and is only determined for Stokes $V$ profiles with a regular shape.

\section{Classification of Stokes $V$ profiles}

In this section we study the shapes of Stokes $V$ profiles produced by the simulations.
In each of the simulation runs, we selected Stokes $V$ profiles with amplitudes above $10^{-3}$ in units of $I_{\mathrm{c}}$. These profiles were then subjected to a Principal Component Analysis (PCA; see, e.g., Rees et al. 2000) similar to that applied by Sánchez Almeida \& Lites (2000) and Khomenko et al. (2003) to the observations of the quiet Sun in visible and IR lines, respectively. The analysis described in this section was performed separately for the Fe I 6302 and $15648 \AA$ lines and each of the snapshots.

The results of the PCA analysis depend on the choice of the database of the example profiles used for the computation of eigenvectors. We constructed this database from the 1000 synthetic Stokes $V$ profiles with the largest amplitudes. Since the different selections of the profiles for the database lead to slightly different classifications, we performed the classification several times, choosing each time a different database. This procedure allowed us to define a statistical error of the classification (for details see Khomenko et al. 2003). The number of profiles belonging to each class together with its standard deviation are given in the corresponding figures (Figs. 3, 4, 6 and 13). All the profiles where normalized to have the same amplitude and signed positive prior to the classification.

\subsection{Stokes $V$ with original spatial resolution.}

We found 16 classes to be sufficient to describe the Stokes $V$ profiles with the original resolution, independent of the magnetic flux in the snapshots. Figure 3 displays examples of Stokes $V$ class profiles of the Fe I $15648 \AA$ line in the $\langle B\rangle=$ $30 \mathrm{G}$ snapshot, as well as their spatial distribution. The profiles of the classes shown in the panels $a$ and $d$ are the most frequent in the simulations, while the profiles in the other panels are chosen to be displayed because they represent interesting examples of the strongly asymmetric and irregular profiles found in our numerical data (see below). The distribution of the class profiles over the snapshot shows a clear correlation with the granulation structure. The classes of the profiles in granules and intergranules are not the same. Figures $3 \mathrm{a}$, b show class profiles typical of intergranules. These profiles are red-shifted and their asymmetries are usually negative (red lobe dominates) or close to zero. The profiles with the highest amplitudes $(a>0.03)$ are located mostly in intergranular lanes, i.e. mainly there where the longitudinal component of the field strength $B_{z}$ is large. Therefore, maps of Stokes $V$ amplitude appear similar to those of $B_{z}$ at the appropriate height (Shelyag 2004). The classes of profiles shown in Figs. 3d and e reside in granular regions. They are blue-shifted and have, on average, positive asymmetry. The profiles in granules are mostly regular. In the case of the original resolution, the irregular profiles are concentrated in the canopy regions, i.e. at the granule/intergranule borders. This follows from the inspection of the map in Fig. 3, where their locations are marked by the red and yellow colors. The average linear size of patches formed by the profiles of the same class (defined as $L_{\text {class }}=\sqrt{A_{\text {class }}}$ with $A_{\text {class }}$ being the area of a patch) is about $200 \mathrm{~km}$. The patches of the profiles belonging to a single class are much smaller in intergranular lanes than in granules, which is not surprising given that lanes are narrower 

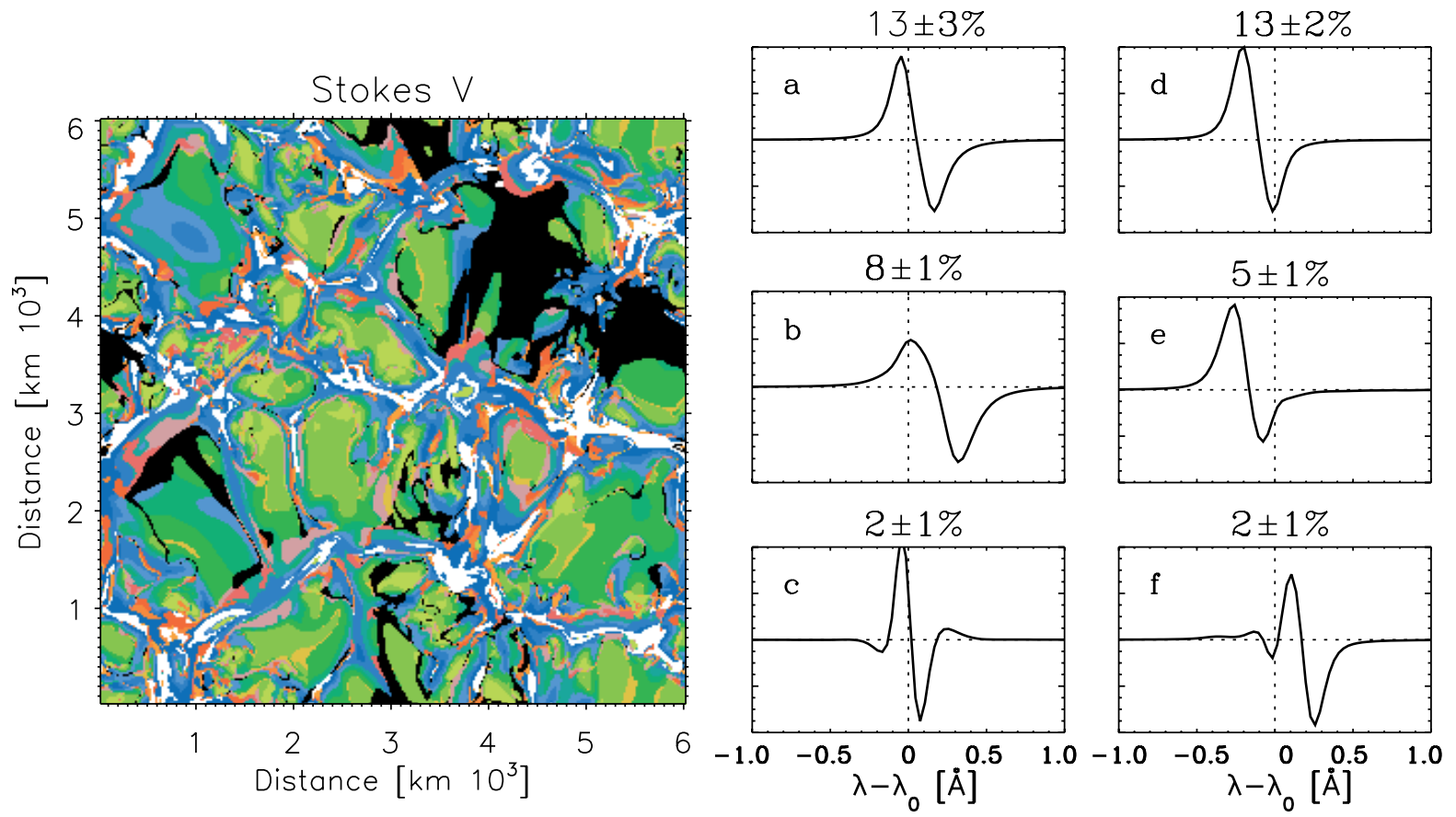

Fig. 3. Left panel: map of the classes of Stokes $V$ profiles of the Fe I $15648 \AA$ line in the $\langle B\rangle=30 \mathrm{G}$ snapshot. Right panels: examples of class profiles (see the discussion in the text). In the left panel green colors mark the locations of the blue-shifted profiles having mostly positive asymmetry (classes d) and e)). Blue and white mark the red-shifted profiles having negative or no asymmetry (classes a) and b)). The yellow and red colors mark the location of the irregularly-shaped profiles (classes $\mathbf{c}$ ) and $\mathbf{f}$ )). The fraction of the profiles belonging to each class is given in percent above each figure. Only profiles with Stokes $V$ amplitudes above $10^{-3}$ are classified.
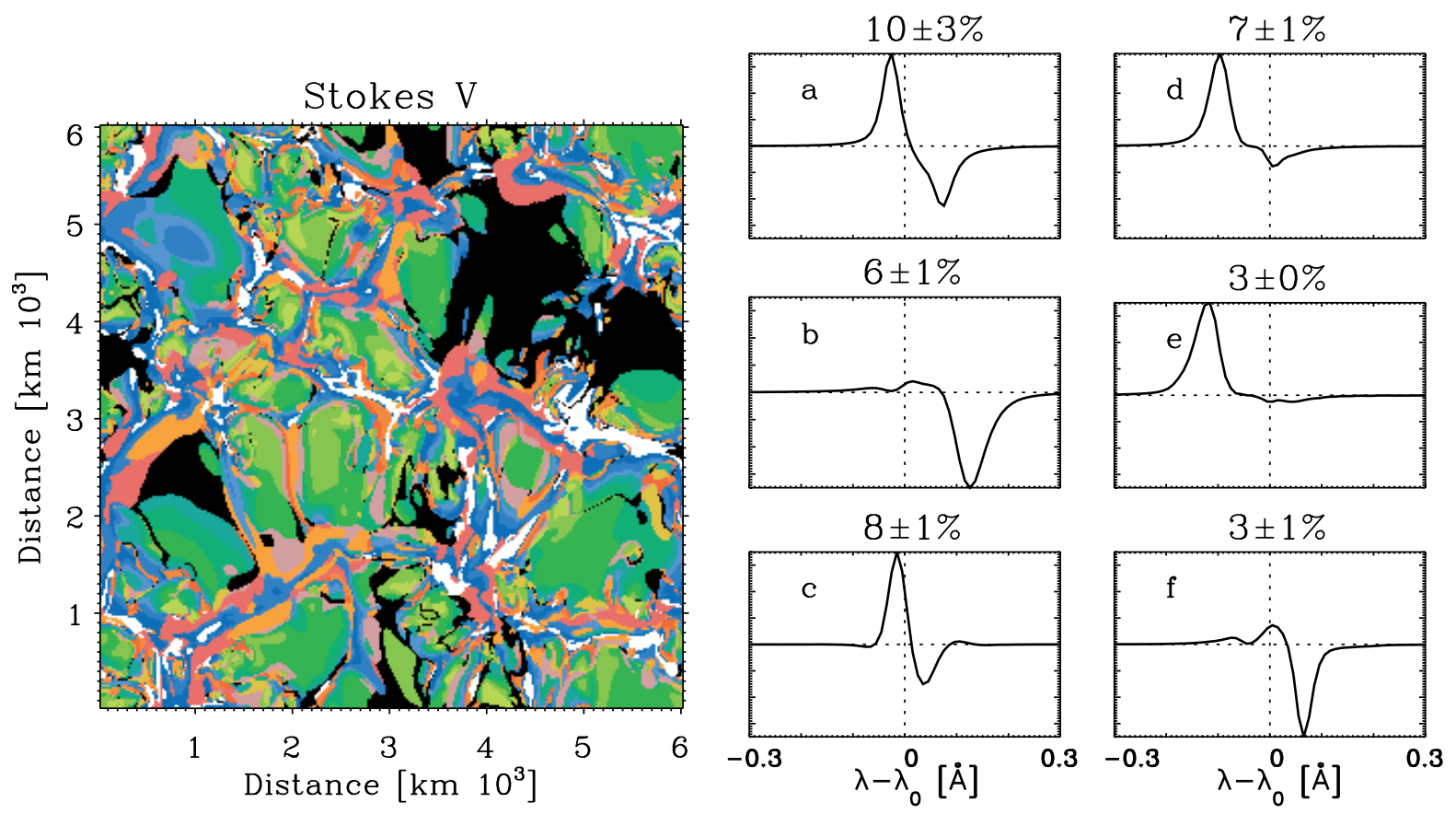

Fig. 4. Left panel: map of the classes of Stokes $V$ profiles of the Fe I $6302 \AA$ line in the $\langle B\rangle=30 \mathrm{G}$ snapshot. The color scheme is the same as in Fig. 3. Right panel: examples of the class profiles.

and more inhomogeneous than granules. The fluctuation spectra of the spatial distribution of the class profiles has significant power down to scales of $40 \mathrm{~km}$.

The results of the PCA classification of the Fe I $6302 \AA$ Stokes $V$ profiles in the $\langle B\rangle=30 \mathrm{G}$ snapshot are shown in Fig. 4. The class profiles of Fe I $6302 \AA$ turned out to be equivalent to the class profiles of the Fe I 15648 A line in Fig. 3 in the sense that the profiles belonging to these classes arise from the same areas in the snapshot. As expected, the asymmetries of the Fe I $6302 \AA$ line profiles are stronger than those of the Fe I $15648 \AA$, since the Zeeman splitting of the former line is smaller. The Zeeman splitting of the Fe I $6302 \AA$ 
Table 2. Fraction of irregular Stokes $V$ profiles of the Fe I 15648 and $6302 \AA$ Aines as a function of the average unsigned magnetic field $\langle B\rangle$ in the simulation snapshots. The last column gives the number of the single-lobed Fe I 6302 A profiles. All profiles are classified irrespective of their amplitudes.

\begin{tabular}{cccc}
\hline \hline$\langle B\rangle[\mathrm{G}]$ & Fe I 15 648 & Fe I 6302 & Single-lobed Fe I 6302 \\
\hline 10 & $33 \%$ & $35 \%$ & $7 \%$ \\
20 & $25 \%$ & $32 \%$ & $6 \%$ \\
30 & $24 \%$ & $33 \%$ & $4 \%$ \\
80 & $23 \%$ & $30 \%$ & $5 \%$ \\
140 & $19 \%$ & $23 \%$ & $2 \%$ \\
\hline
\end{tabular}

line is of the order of its Doppler width and hence is close to the typical difference in velocity over the line formation height (Grossmann-Doerth et al. 1989). However, as follows from the comparison of the maps of the class profiles in Fig. 3 and Fig. 4 (the maps have the same color coding) the sense of the asymmetries of both spectral lines arising from the same pixels is similar. The fraction of irregular profiles is larger in the visible spectral range. These profiles are still found in the canopy regions, but now occupy larger areas. The profiles most frequently obtained in simulations have positive asymmetry. The prevalence of the positive asymmetry is a robust characteristic of the observed profiles of this line in the plage, network and inter-network solar regions near disc center (Martínez Pillet et al. 1997; Sigwarth et al. 1999). This indicates that the distributions of the velocity, magnetic field and other parameters in the simulation have characteristics close to the real Sun, although we need to bear in mind that the spatial resolution of the simulations and the numerical data are rather different.

The class profiles for the other snapshots are, in general, similar. The main difference between the snapshots is the number of irregular profiles. Table 2 presents the fraction of the irregular profiles in the snapshots with different flux levels. Note, that unlike the case of the PCA classification presented above in Figs. 3 and 4, no threshold was applied to the profiles when preparing the data given in Table 2. According to this table, the fraction of irregular profiles increases with decreasing magnetic flux. The latter is true in both spectral regions, with the number of irregular profiles in the visible being always larger. A similar dependence on the average field strength was also seen in observational data (Sánchez Almeida \& Lites 2000; Sigwarth 2001; Socas-Navarro \& Sánchez Almeida 2002; Khomenko et al. 2003). However, a quantitative comparison of the fraction of irregular profiles is problematic due to the large difference in the spatial resolution and to the absence of a threshold applied to the profiles in Table 2.

The classification of the Fe I 6302 Stokes $V$ profiles revealed classes having only one (blue of red) significant lobe (such as, e.g., classes $b$ and $e$ in Fig. 4). In the absence of noise, we defined a profile as single-lobed if the amplitude of the other lobe was lower than $15 \%$ of the first one. The fraction of the single-lobed profiles also increases with decreasing flux and is minimal for the $\langle B\rangle=140 \mathrm{G}$ snapshot. Single-lobed profiles are absent in the infrared.

Without going into details, we briefly discuss the formation of irregular $V$ profiles in the simulations. In the case of the original spatial resolution, the irregular profiles are produced by the changes of the polarity of the field along the line-ofsight in the presence of velocity gradients (see Ploner et al. 2001 , who presented such a discussion based on the 2D simulations of Gadun et al. 2001). We have counted the number of times the vertical magnetic field component crosses the $B_{z}=0$ line in all the pixels where the spectra were calculated. Within a given snapshot, polarity changes along the line-ofsight are less frequent in granules (where $B_{z}$ is small) and in the centers of intergranular lanes (where $B_{z}$ is strongest). The $B_{z}$ sign changes are most frequent at the lines-of-sight located at granular-intergranular borders. This produces a larger number of the irregular profiles there. The average number of crossings (i.e. sign changes) over all rays appeared to decrease from 1.4 for the $\langle B\rangle=10 \mathrm{G}$ snapshot to 0.87 for the $\langle B\rangle=140 \mathrm{G}$ snapshot. This explains the trend towards a larger fraction of irregular profiles in snapshots with lower $\langle B\rangle$ (see Table 2).

Figure 5 gives an illustrative example of the formation of a typical irregular profile of class $c$ from Figs. 3 and 4. It shows a two-dimensional cut through the magnetic feature. The Fe I 15648 and $6302 \AA$ line profiles shown in the middle panel emerge from the region close to a granular border. The vertical magnetic field is very weak in these regions. It changes polarity twice within the height range of line formation. At the same time, a strong velocity gradient exists, that displaces the contributions to the line profile from different optical depths in wavelength relative to each other. The combined action of these two agents results in an irregular Stokes $V$ profile having 4 lobes. Hence it appears to be mainly the gradient in the inclination angle of the magnetic vector (combined with a LOS velocity gradient), which is responsible for producing the irregular $V$ profile, rather than changes in the field strength. Thus, at least for this set of profiles, the situation is similar to the formation of irregular profiles in sunspot penumbrae (Sánchez Almeida \& Lites 1992; Solanki \& Montavon 1993). For the production of the area asymmetry of Stokes $V$ another ingredient is also required, line saturation introduced by the radiative transfer through the atmosphere (see Solanki 1993; Steiner 2000, for illustration of this process).

\subsection{Stokes $V$ with reduced resolution}

The line profiles discussed above cannot be compared directly with observations due to the difference in the spatial resolution. To make such a comparison possible, the original profiles were smeared as described in Sect. 3.1. The parameters of the smearing function were chosen to make the spatial resolution and continuum contrast $\delta I_{\mathrm{c}}$ compatible with the values typical of high-resolution observations. The telescope diameter for the computation of the Airy point-spread function was taken to be $70 \mathrm{~cm}$, which corresponds to the diameter of the Vacuum Tower Telescope at the Observatorio del Teide, Tenerife. After the convolution, the spectra at $6300 \AA$ have $\delta I_{\mathrm{c}}=7 \%$ and spatial resolution 0.5 , while the spectra at $1.56 \mu \mathrm{m}$ have $\delta I_{\mathrm{c}}=3 \%$ and a resolution of $0 . ' 8$. We also averaged 4 by 4 original pixels together, so that the new effective pixel size is $80 \mathrm{~km}$. 

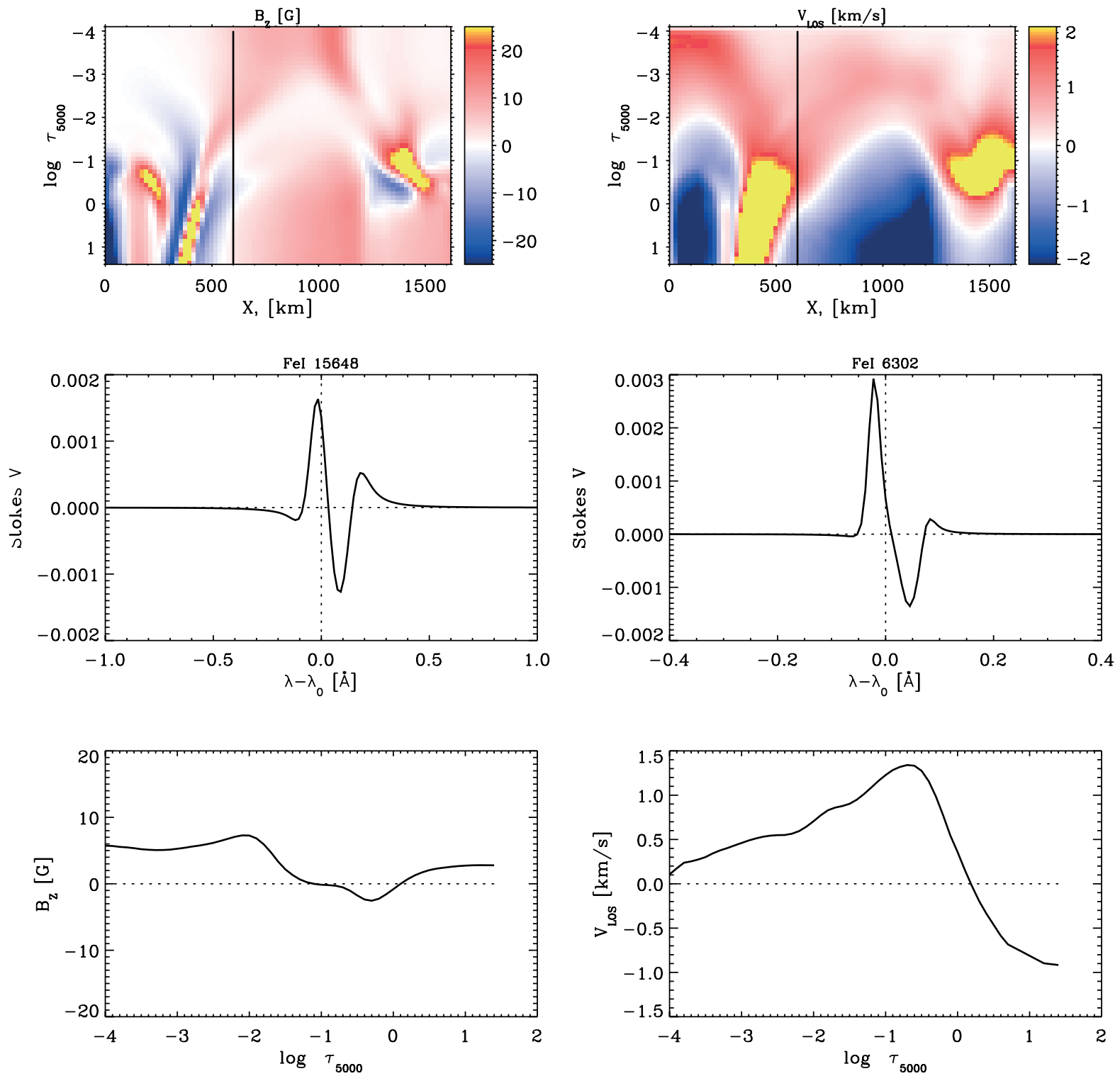

Fig. 5. Example of the formation of the irregular Stokes $V$ profile belonging to the classes shown in Figs. 3c and 4c. Top panels: distributions of the vertical magnetic field and velocity with optical depth and horizontal distance. The vertical line marks the ray along which the Fe I $15648 \AA$ and $6302 \AA$ profiles displayed in the middle panels are formed. Bottom panels: the corresponding magnetic field and velocity profiles on the optical depth scale.

The results of the PCA classification of the Fe I $15648 \AA$ Stokes $V$ profiles in the $\langle B\rangle=30 \mathrm{G}$ snapshot with reduced resolution are presented in Fig. 6 . Similar to the case of the original resolution, no noise was added to the profiles, but we classify all the profiles with amplitudes above a threshold of $10^{-3}$. Despite the spatial smearing, the Stokes $V$ with the largest amplitudes (above 0.01) are located in intergranular lanes, in agreement with the unsmeared case (Sect. 4.1). However, the class profile distribution as a whole does now follow the granulation pattern any more. No clear distinction can be made between the class profiles in granules and intergranular lanes. The irregular profiles are now located in between the opposite polarity areas in addition to the edges of the strong-field patches. The patches formed by the profiles of the same class have become larger as compared to the unsmeared case. Their average size is close to the spatial resolution, i.e. about $600-700 \mathrm{~km}$ (as also found in the observations by Khomenko et al. 2003). The fact that many of the irregular profiles are now located between opposite magnetic polarities suggests that horizontal magnetic gradients, i.e. changes in magnetic polarity perpendicular to the line of sight, are now an important, possibly even dominant cause of irregular profiles.

The seeing causes the profiles to become broader and more asymmetric. The profiles most frequently obtained from the simulations after smearing all show positive asymmetry (see Figs. 6a, b, d). Similar classes of profiles can also be found in observations. We have plotted some of the class profiles from the observations of the quiet Sun in the Fe I $15648 \AA$ line (Khomenko et al. 2003) over the class profiles given by the simulations. For most of the regular class profiles deduced from 


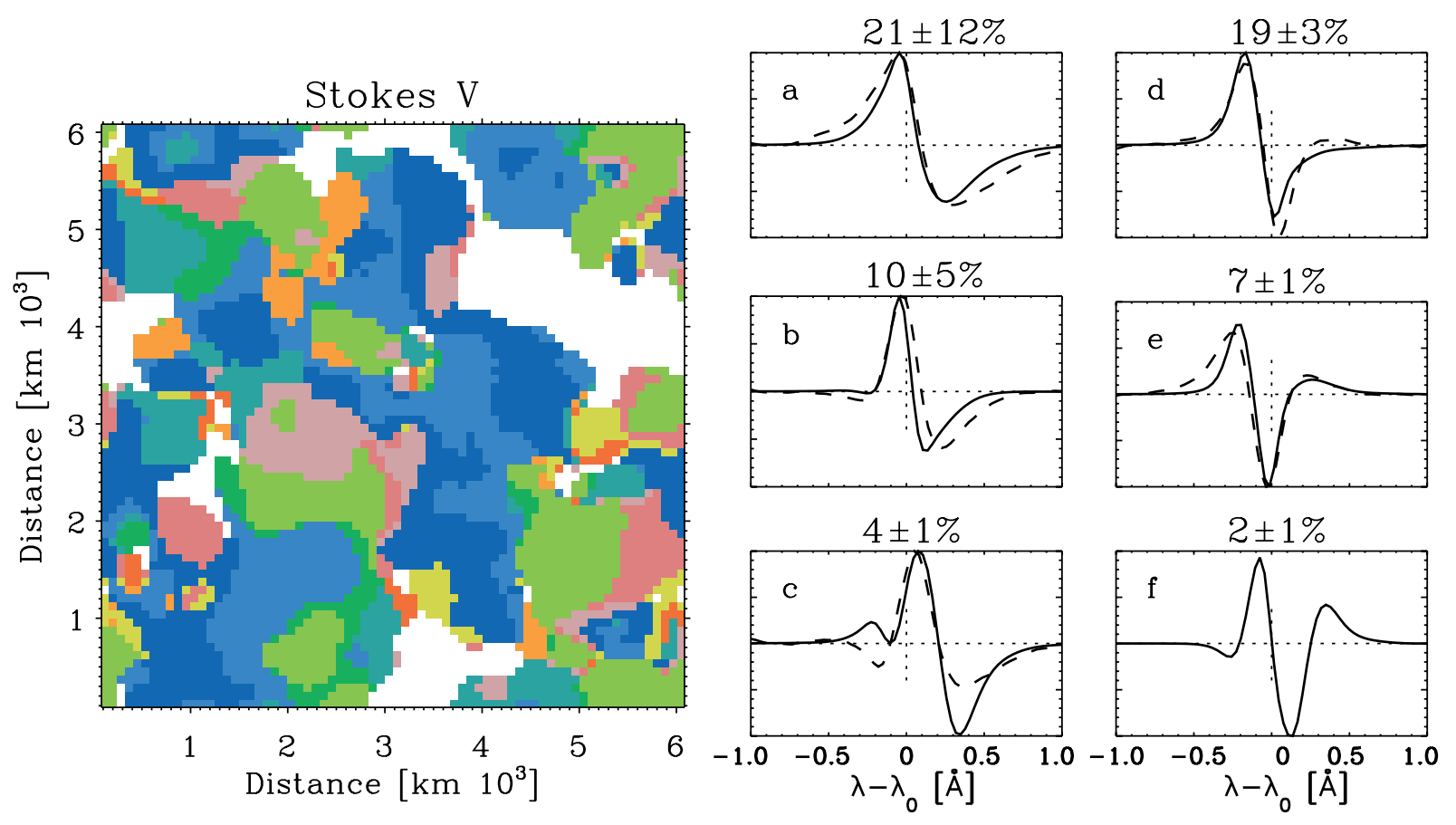

Fig. 6. Left panel: map of the classes of Stokes $V$ profiles of the Fe I $15648 \AA$ line in the $\langle B\rangle=30 \mathrm{G}$ snapshot. The profiles were smeared to have a resolution of $0^{\prime \prime} 8$ and a contrast $\delta I_{\mathrm{c}}=3 \%$. Only profiles with amplitudes above $10^{-3}$ are classified. The color coding is the same as in Fig. 3. Right panel: examples of the class profiles. Solid line: class profiles from the simulations; dashed line: class profiles from the quiet Sun observations in the infrared (Khomenko et al. 2003).

the simulations we were able to find an equivalent class profile in the observations. Some irregular-shaped class profiles also have their equivalents (e.g., classes $e$ and possibly $c$ in Fig. 6). However, some of the simulated profile shapes are not found in observations (see, for example, class $f$ with 4 lobes). This does not itself imply fundamental discrepancy between simulations and observations since one or both weak side lobes of the 4-lobed profiles can have amplitudes below the noise and such profiles can be assigned to the 3-lobed class $e$ or even classified as normal. This interpretation is supported by the fact that the 4-lobed profiles generally have a low amplitude. In one respect there is a significant discrepancy: the observed class profiles are often broader than those resulting from the simulations (compare classes $a$ and $b$ ). The presence of broader profiles in the observations can have different causes. One possibility is that class profiles given by the PCA technique represent the average over all profiles belonging to a class. This averaging broadens the class profile. In the observations some 8000 profiles were classified while in the simulations only half this number was employed. Also it must be noted that the center wavelength of the Fe I 15648 line is not precisely determined from the observations and may contain a residual blueshift (see Khomenko et al. 2003) while in both simulations and observations it can be affected by the oscillations (see Sect. 7). Thus, a wavelength shift is possible between the simulated and the observed class profiles.

The number of the irregular profiles in the simulations depends on the criteria applied for their selection. We performed two independent classifications. In the first case, we classified all the profiles irrespective of their amplitudes. Thus, this classification provides an estimate of the "true" number of the
Table 3. Fraction of irregular Stokes $V$ profiles of the Fe I 15648 and $6302 \AA$ A lines with reduced resolution as a function of $\langle B\rangle$. All profiles irrespective of their amplitudes are classified.

\begin{tabular}{ccc}
\hline \hline$\langle B\rangle[\mathrm{G}]$ & Fe I 15648 & Fe I 6302 \\
\hline 10 & $36 \%$ & $57 \%$ \\
20 & $39 \%$ & $60 \%$ \\
30 & $41 \%$ & $53 \%$ \\
80 & $38 \%$ & $51 \%$ \\
140 & $26 \%$ & $31 \%$ \\
\hline
\end{tabular}

irregular profiles. In the second case, we added noise to the profiles prior to the classification and applied a threshold to select the profiles with amplitudes above the noise. The noise not only changes the profiles amplitudes, but also their classification by often leaving the weaker lobes of the irregular profiles undetected. The results of the second classification can be compared with observations. The results of both classifications are given in Tables 3 (all profiles) and 4 (noisy profiles). Note, that both classifications are different from the one presented in Fig. 6 where only profiles with amplitudes about $10^{-3}$ were considered and no noise was added.

Table 3 compared with Table 2 shows that the spatial smearing has increased the number of the irregular profiles in both spectral ranges by a factor of 1.5 (on average). Since the spatial distribution of the irregular profiles also has changed (see Fig. 6), it means that most of the irregular profiles are due to horizontal variations of the parameters. The information about their variations along the line-of-sight is masked. This could mean that, in the observations with a typical resolution of $0.5-1^{\prime \prime}$, the presence of the irregular profiles reflects mainly 
Table 4. Fraction of irregular Stokes $V$ profiles of the Fe I 15648 and $6302 \AA$ lines with reduced resolution as a function of $\langle B\rangle$. Only the profiles with amplitudes 3 times above the noise of $3 \times 10^{-4} I_{\mathrm{c}}$ are considered. The last two columns give the fraction of profiles above the threshold in the infrared and the visible.

\begin{tabular}{ccccc}
\hline \hline$\langle B\rangle[\mathrm{G}]$ & Fe I 15 648 & Fe I 6302 & $N_{15648}$ & $N_{6302}$ \\
\hline 10 & $22 \%$ & $21 \%$ & $36 \%$ & $31 \%$ \\
20 & $29 \%$ & $30 \%$ & $64 \%$ & $54 \%$ \\
30 & $26 \%$ & $29 \%$ & $86 \%$ & $88 \%$ \\
80 & $19 \%$ & $28 \%$ & $99 \%$ & $100 \%$ \\
140 & $16 \%$ & $17 \%$ & $100 \%$ & $100 \%$ \\
\hline
\end{tabular}

the horizontal distribution and mixing of the field rather than its vertical structure.

After adding the noise and introducing a threshold, the fraction of the profiles, classified as irregular, decreases (Table 4). The numbers given in the table are calculated in percent from the total number of analyzed profiles above the threshold (see last two columns in Table 4). The fraction of irregular profiles now has on average halved compared to Table 3 . This is because on average the irregular $V$ profiles have lower amplitudes. In all the considered snapshots, irregular profiles have amplitudes about 2.5 lower than normal profiles in the infrared and 3.5 times lower in the visible. On the other hand, the smallest lobes lie below the noise and are often not identified as such, so that many of these profiles are classified as regular. The irregular profiles are less abundant in the large-flux case of $\langle B\rangle=140 \mathrm{G}$ and also in the lowest-flux case of $10 \mathrm{G}$. In the latter case, due to the weakness of the fields most of the irregular profiles remain below the noise. The variety of profiles shapes has decreased. The most frequent type of irregular profile is 3-lobed. The fraction of irregular profiles is now similar in the IR and in the visible. For the snapshots with $\langle B\rangle 10-80 \mathrm{G}$, this fraction is close to that observed in the quiet Sun (Sánchez Almeida \& Lites 2000; Socas-Navarro \& Sánchez Almeida 2002; Khomenko et al. 2003). The horizontal distribution of the irregular profiles suggests that horizontal gradients are the main cause of such profiles, which is similar to the case without noise.

The classification of the profiles with this noise level is relatively stable. We found that the fraction of irregular profiles does not change significantly in the classifications with and without noise if the same threshold is applied. The only important change between these cases is the relative distribution of 3-lobed and 4-lobed irregular profiles. After adding noise, the latter are not identified anymore in the lower flux snapshots and only remain in the classifications of the 80 and $140 \mathrm{G}$ snapshots.

After smearing, the fraction of single-lobed profiles significantly decreases. Their fraction remains within $0.5-1 \%$ in all the considered snapshots and for both spectral lines (irrespectively on whether or not noise is added). It was reported by Grossmann-Doerth et al. (2000) that single-lobe profiles make up some $3 \%$ of the $35 \%$ of all profiles with amplitudes above the threshold in the quiet solar region (i.e. about $1 \%$ of the total field of view). This number is consistent with the value deduced from the simulations.

\section{Amplitudes of Stokes $V$}

Figure 7 displays the distributions of the amplitudes of Stokes $V$ profiles at the original resolution. It shows the distributions for a weak-flux snapshot $(\langle B\rangle=20 \mathrm{G})$ and a strong flux one $(\langle B\rangle=140 \mathrm{G})$. We measured the amplitudes as the maximum absolute values of Stokes $V$ in each individual spatial point. The mean values of the amplitudes are indicated by the arrows. The range of the amplitudes is very large, varying by $4-5$ orders of magnitude. Not only does the mean value increase with $\langle B\rangle$, but the shape of the distribution also changes.

Now, we expect the average amplitude to increase with increasing flux. However, the increase is not linear due to Zeeman saturation (e.g. Stenflo 1973). Without spatial smearing, the amplitudes of Stokes $V$ profiles formed in weak-field areas are proportional to the magnetic field strength in the pixel, while in strong-field pixels the amplitude of the Stokes $V$ is independent of the field strength and the separation between the lobes is affected. The number of pixels where the weak-field approximation breaks down is larger in the large-flux snapshots. Since the weak-field approximation breaks down at lower $B$ values for the IR lines than for the visible lines, the average amplitude of the visible lines becomes larger. Indeed, Fig. 7 shows that in the $\langle B\rangle=20 \mathrm{G}$ snapshot the average amplitudes of Stokes $V$ are approximately equal in both spectral ranges. In the $\langle B\rangle=140 \mathrm{G}$ case the distribution saturates at approximately 0.1 for the IR and at 0.3 for the visible (in units of $I_{\mathrm{c}}$ ), so that the average amplitude becomes 1.7 times larger in the visible. Thermal effects may, of course, also play a role in determining the shape of the distribution.

The Stokes $V$ amplitude distribution for reduced spatial resolution is given in Fig. 8. The spectra are smeared and have the same resolution and contrast as used in Sect. 4.2. No noise was added to the profiles.

Reducing the resolution causes two effects. Firstly, the redistribution of the signal leads to the reduction of the amplitudes of the strongest profiles accompanied by an increase of the amplitudes of the weak profiles in the neighboring pixels. Thus, the strongest and the weakest signals disappear (compare Fig. 7a with Fig. 8b). Secondly, the average Stokes $V$ amplitude in the box decreases. As the average amplitude has decreased, a part of the flux contained in the box can remain undetected (since $\langle B\rangle$ is proportional to Stokes $V$ amplitude in the weakfield regime).

The decrease of the average amplitude can be caused by several effects. Up to half of the average original amplitude can be lost by averaging profiles with significantly different displacements and splittings like those in granules and intergranules. Another source of losses is cancellation of elements with different polarities. While the first effect is always present in granulation, the second one will depend on the spatial mixing of the field with opposite polarities. In the case of the simulations, after reducing the resolution from $20 \mathrm{~km}$ to about 500-600 km, the average polarization signal becomes almost 3 times lower in both lines in all considered snapshots (compare the positions of the arrows in Figs. 7 and 8). It means that up to $2 / 3$ of the original flux can remain undetected in typical 

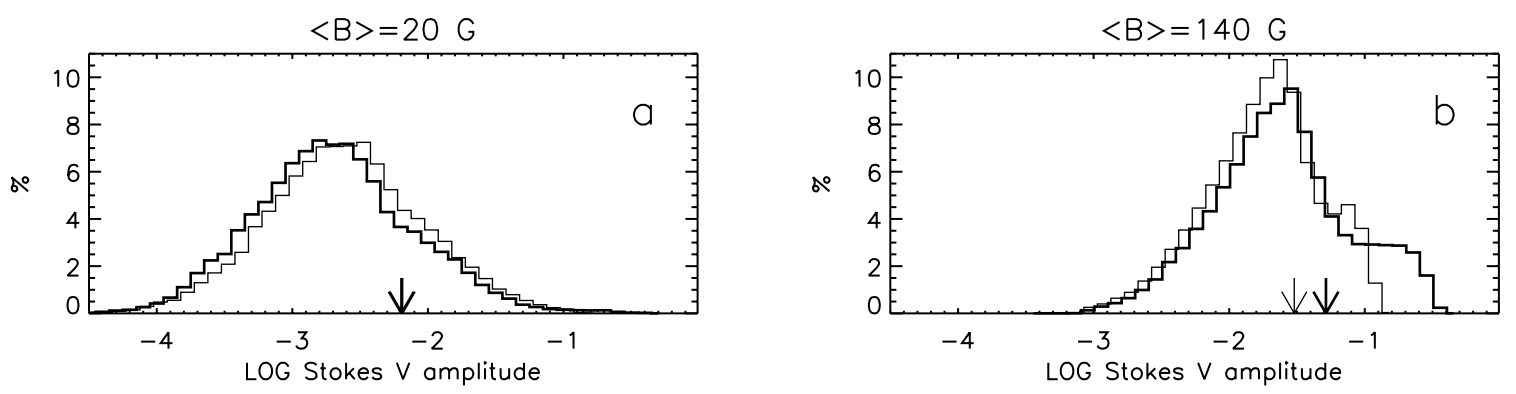

Fig. 7. Histogram of the amplitudes of Stokes $V$ profiles with original resolution for the $\langle B\rangle=20$ and $140 \mathrm{G}$ snapshots (panels a) and b), respectively). Thick line: Fe I $6302 \AA$, thin line: Fe I $15648 \AA$. The arrows indicate the mean values in both cases.
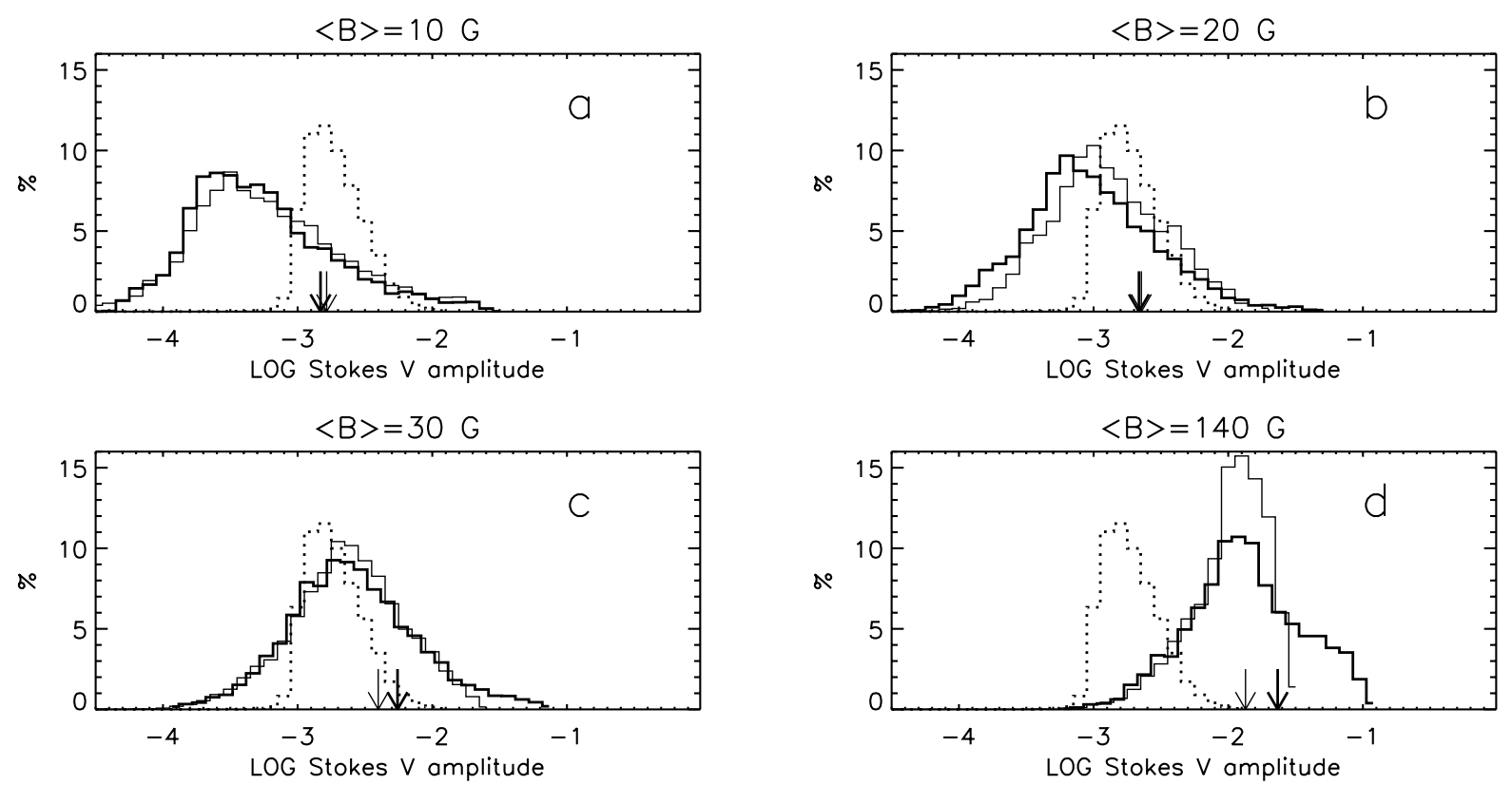

Fig. 8. Histogram of the amplitudes of Stokes $V$ profiles with reduced resolution for the $\langle B\rangle=10,20,30$ and $140 \mathrm{G}$ snapshots (panels a), b), c) and d), respectively). Thick line: Fe I 6302; thin line: Fe I 15 648; dotted line: observations in the infrared taken from Khomenko et al. (2003).

observations if one uses Stokes $V$ amplitudes as a measure of the magnetic flux.

The relation between the amplitudes of the Fe I 6302 and $15648 \AA$ lines in the snapshots with different $\langle B\rangle$ appear to be relatively independent of spatial resolution (compare the positions of the arrows in Figs. $7 \mathrm{a}$ with $8 \mathrm{~b}$ and Figs. $7 \mathrm{~b}$ with $8 \mathrm{~d}$ ). The average amplitude is slightly larger in the infrared for $\langle B\rangle=10 \mathrm{G}$. In the $\langle B\rangle=20 \mathrm{G}$ snapshot, the amplitudes are approximately equal in the visible and infrared. But in all the other snapshots the polarization signal in Fe I $6302 \AA$ is stronger. This has to do with the increasing fraction of strong field pixels with increasing $\langle B\rangle$ and the more prominent Zeeman saturation of the Fe I $15648 \AA$ compared with Fe I $6302 \AA$.

How do the amplitudes compare with the observations? In Fig. 8, we also plot the observed Fe I $15648 \AA$ Stokes $V$ amplitude distribution adapted from the observations of Khomenko et al. (2003) (the resolution of the observed and numerical spectra are close). In these observations, the noise level did not allow signals below $10^{-3} I_{\mathrm{c}}$ to be reliably identified, which is responsible for the rapid drop-off at $10^{-3}$ of the observed histogram. The comparison reveals that the amplitudes given by the simulations are too weak in the $10 \mathrm{G}$ case and too strong for the $30 \mathrm{G}$ case. In the $20 \mathrm{G}$ case, however, the right part of the observed histogram is well approximated by the theoretical distribution. The difference in the left part is due to the fact that no threshold was applied to the profiles resulting from the simulations. This indicates that the "true" average unsigned magnetic field strength contained in the inter-network region in the observations of Khomenko et al. (2003) was close to $20 \mathrm{G}$. Note that the fraction of the area covered by the signals above the $10^{-3}$ threshold is similar in the observations and the simulations with $\langle B\rangle=20 \mathrm{G}$ (see Table 4). These results are similar to those obtained by Khomenko et al. (2005) by comparing these simulations with simultaneous measurements made with TIP (Martínez Pillet et al. 1999) and POLIS (Schmidt et al. 2000).

The average magnetic field of $20 \mathrm{G}$ corresponds to an average longitudinal flux $\left\langle\left|B_{z}\right|\right\rangle$ of $11 \mathrm{G}$ at $\log \tau=-1$ and $15 \mathrm{G}$ at $\log \tau=0$ in the simulation box. Thus, if the amplitude of Fe I 15648 line is used as a measure of the flux and a signal is detected in $100 \%$ of the points (i.e. infinitely large polarimetric sensitivity of observations) then values of $\left\langle\left|B_{z}\right|\right\rangle$ about 

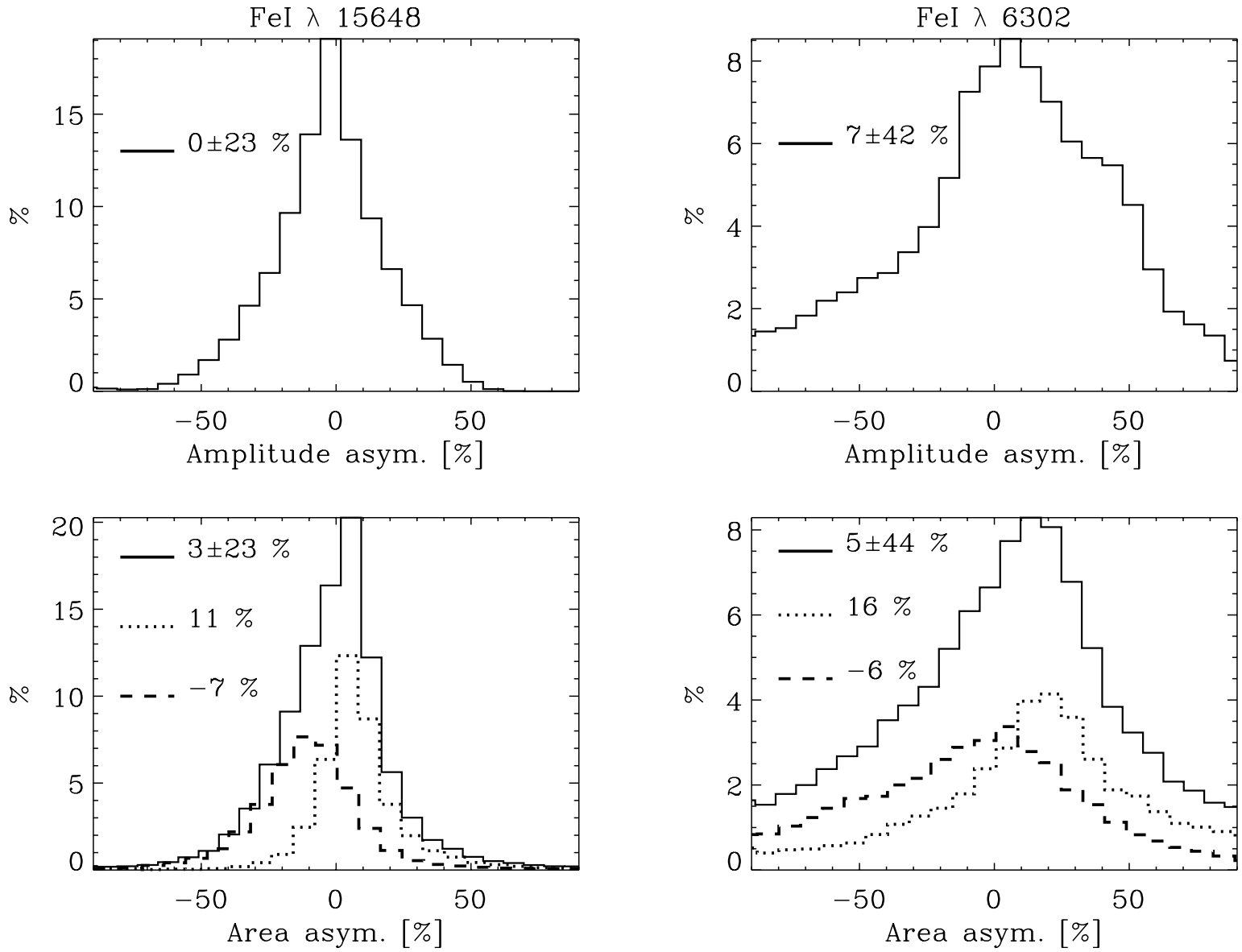

Fig. 9. Histograms of the amplitude (top) and area (bottom) asymmetries of the Stokes $V$ profiles in the $\langle B\rangle=20 \mathrm{G}$ snapshot at its original resolution. Sold line: all regular-shaped profiles with amplitudes above $10^{-3}$. Dashed line: only profiles in downflowing regions. Dotted line: only profiles in upflowing regions. Left panels: Fe I $15648 \AA$, right panels: Fe I $6302 \AA$. The mean values together with standard deviations corresponding to each curve are given in each panel.

4-5 $\mathrm{G}$ would be obtained. The value reported by Khomenko et al. (2003) was $8 \mathrm{G}$. Due to the noise in observations only the highest-amplitude signals were detected in some $60 \%$ of the observed area. The magnetic field averaged over these points is larger than the average over the whole area. However, note that a technique different from a simple measure of amplitudes was applied by these authors to infer the magnetic flux.

\section{Asymmetries}

Histograms of the amplitude and area asymmetries of the Stokes $V$ profiles are displayed in Fig. 9 for the $\langle B\rangle=20 \mathrm{G}$ snapshot at the original resolution (i.e. $20 \mathrm{~km}$ grid size). The difference between the distributions for the snapshots with different magnetic flux is rather weak. The mean values of the amplitude asymmetries of the profiles with original resolution remain almost independent of the flux. Stokes $V$ profiles of the Fe I $15648 \AA$ line show mild amplitude asymmetries with a mean value close to zero. The area asymmetries are, on average, positive (see left panels of Fig. 9). Mean values of the area asymmetries increase slightly with increasing flux from 2 to $5 \%$. In the case of the Fe I $6302 \AA$ line, profiles having positive $\delta a$ and $\delta A$ dominate the distribution. The amplitude asymmetry of the Fe I $6302 \AA$ remains around 7-9\% independently of the flux and the area asymmetry increases from 5 to $9 \%$ over the considered flux interval.

The range of asymmetry of the visible line is much larger than of the infrared one. For both lines, the range of asymmetry variations (measured as a standard deviation) is $3-7 \%$ lower in the $140 \mathrm{G}$ snapshot than in the rest of the snapshots.

Profiles in the upflowing regions are characterized on average by positive area asymmetries (blue lobe dominates), while the profiles in the downflowing regions have negative asymmetries, although these are on average weaker. A similar behaviour was first noted in 2D simulations by Sheminova (2003). Recently Socas-Navarro et al. (2004) studied weak polarization signals in Fe I $6302 \AA$ observed in an inter-network region and showed that on average over granules Stokes $V$ profile are characterized by a strong positive asymmetry, while in intergranules the asymmetry is negative and weaker. Thus, the simulations are in qualitative agreement with these observations, despite the difference in the spatial resolution.

Such a distribution of asymmetries can be traced back to the original synthesis and to the line-of-sight gradients of the velocity, magnetic field strength $B$ and its inclination $\gamma$. According to Solanki \& Pahlke (1988) and Sánchez Almeida et al. (1989), the sign of the area asymmetry $\delta A$ produced by the magnetic field gradient is given by the sign of the 

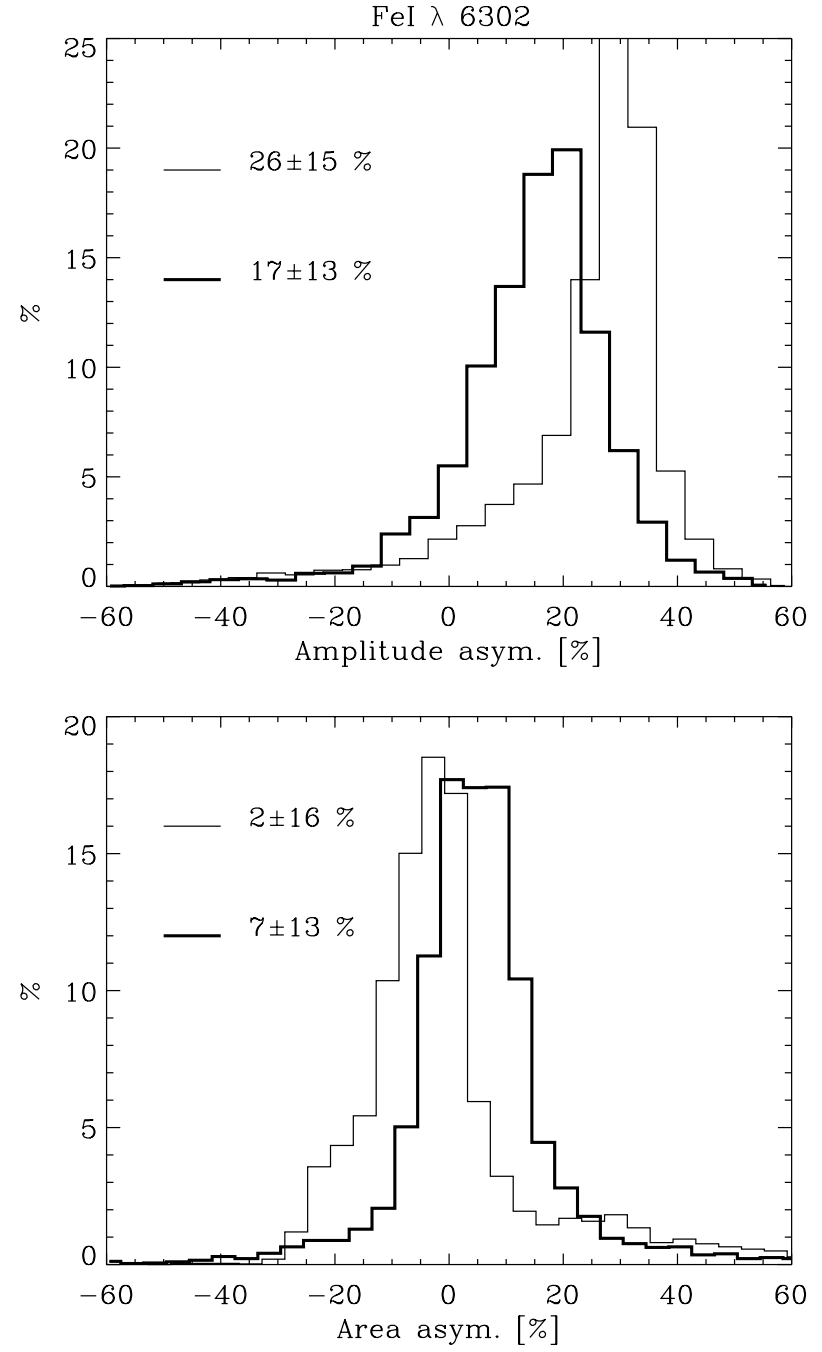

Fig. 10. Histograms of the amplitude (top) and area (bottom) asymmetries of the Fe I $6302 \AA$ Stokes $V$ profiles at reduced resolution. All regular-shaped profiles with amplitudes above $10^{-3}$ are considered. Thick line: $\langle B\rangle=140 \mathrm{G}$; thin line: $\langle B\rangle=30 \mathrm{G}$.

anti-correlation between the gradients of magnetic field strength $|B|$ and velocity $v$ in the heights of the line formation. While the sign of $\delta A$ produced by the gradient of the inclination is given by the sign of the anti-correlation between $|\cos \gamma|$ and $v$ (Solanki \& Montavon 1993). The magnetic and velocity gradients in the simulations of relevance for the production of the asymmetries has been investigated by Shelyag et al. (2005). The gradient indeed shows a pattern that agrees with the expectation from the line asymmetries.

Distributions of the amplitude $(\delta a)$ and area $(\delta A)$ asymmetries of the smeared profiles at roughly $1^{\prime \prime}$ resolution are presented in Figs. 10 and 11 for $\langle B\rangle=140 \mathrm{G}$ and $30 \mathrm{G}$. The shape of the histograms and the mean value of the asymmetry significantly depend on $\langle B\rangle$. We note the following points.

Firstly, the histograms are narrower after smearing, with the width of the distribution of the amplitude asymmetry $\delta a$ now being similar for the IR and visible lines. Spatial smearing leads to a strong reduction in the number of regular $V$ profiles with the strongest asymmetries (the number of irregular profiles increases, however, see Tables 2 and 3).
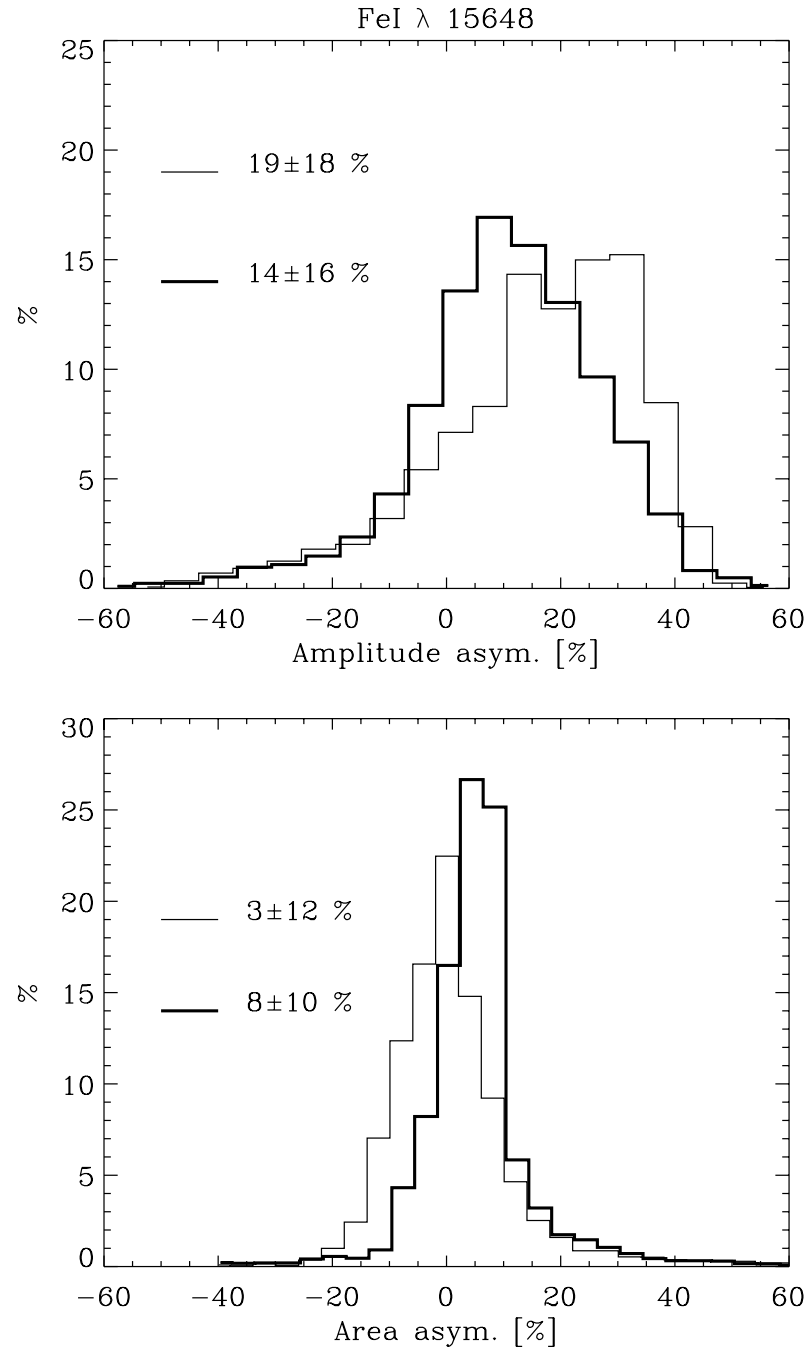

Fig. 11. Same as Fig. 10 but for Fe I 15648 A.

Secondly, the $\delta a$ and $\delta A$ histograms for the smeared profiles are shifted toward positive values. The average $\overline{\delta a}$ values are larger than the values for the non-smeared profiles. The $\overline{\delta a}$ decreases from 27 to $17 \%$ (Fe I 6302) and from 20 to $14 \%$ (Fe I 15 468) as the average magnetic field strength increases from 10 to $140 \mathrm{G}$ (note that Figs. 10-11 show histograms for the 30 and $140 \mathrm{G}$ snapshots as illustrative examples). The area asymmetry $\overline{\delta A}$ shows the opposite behaviour. It increases from negative $(-4 \%)$ to positive ( $8 \%$ ) values over the same $\langle B\rangle$ interval (the values are similar for both spectral lines). This dependence of the area asymmetry on the flux probably comes from the original synthesis since already the original resolution profiles display a similar trend. The trend in the amplitude asymmetry (that is present only under reduced resolution) could be due to the difference in horizontal gradients between the snapshots with the different flux level.

How do the asymmetries of the synthetic lines compare with observations? The observations of the quiet Sun (mainly network fields) in the visible lines at $6300 \AA$ give values of $\delta a=15 \%$ and $\delta A=6 \%$ (Sigwarth et al. 1999). Both these values decrease in the case of plages and active regions to $\delta a=9-10 \%$ and $\delta A=1-3 \%$ (Martínez Pillet et al. 1997; 

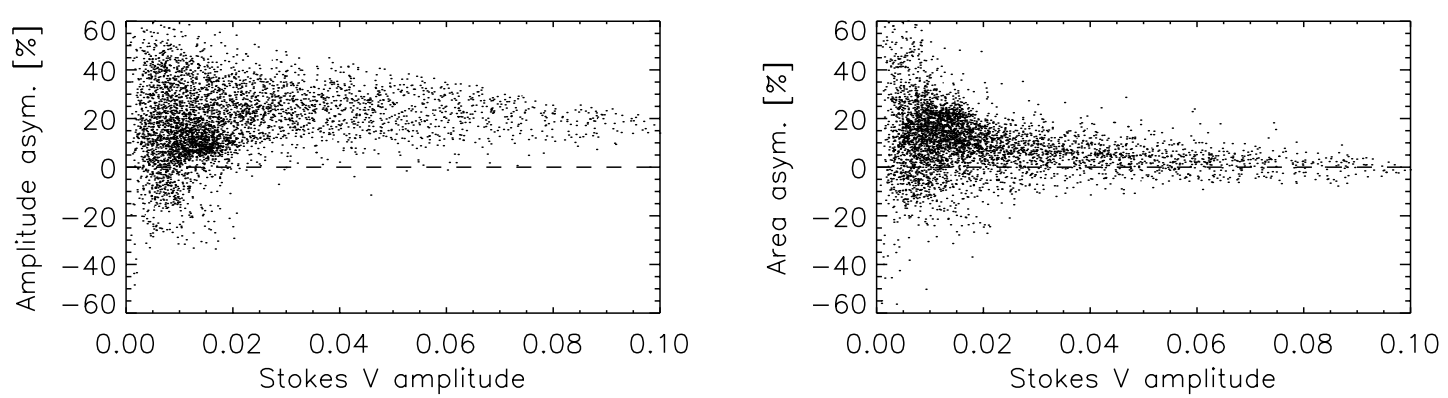

Fig. 12. Scatter plots of the Fe I $6302 \AA$ Stokes $V$ amplitude asymmetry (left panel) and area asymmetry (right panel) as a function of Stokes $V$ amplitude in the $\langle B\rangle=140 \mathrm{G}$ snapshot.

Sigwarth et al. 1999). The quiet Sun's inter-network magnetic field observed in the infrared at $1.56 \mu \mathrm{m}$ displays $V$ profiles with amplitude and area asymmetries of $15 \%$ and $7 \%$, respectively (Khomenko et al. 2003). Thus, both amplitude and area asymmetries in Fig. 10 in the $140 \mathrm{G}$ case agree with the network observations in the same line. However, the agreement with the inter-network observations is not of equally good quality. In the lower-flux case of $30 \mathrm{G}$ (thin line in Fig. 11), the amplitude asymmetry of Fe I $15648 \AA$ is too large and the area asymmetry is too small as compared to the observations of Khomenko et al. (2003).

Finally, at roughly $1^{\prime \prime}$ resolution the asymmetries in granules and intergranules are not the same. Figure 12 shows the dependence of the asymmetries on the Stokes $V$ amplitudes for the Fe I $6302 \AA$ and $\langle B\rangle=140 \mathrm{G}$. The lower-amplitude profiles in granules have predominantly positive area asymmetry, while higher-amplitude profiles in intergranules have area asymmetry close to zero. A simple average of the asymmetry values in these regions is about $\delta A=10 \%$ in granules and $\pm 1 \%$ in intergranules, irrespective of the magnetic flux in the considered snapshots. This tendency is a result of the original distribution of the asymmetries due to vertical gradients (Fig. 9) and is in agreement with the observations of Socas-Navarro et al. (2004). The amplitude asymmetry shows a more complex behaviour: the asymmetry of the large-amplitude profiles is positive, although decreasing with $V$ amplitude, while the scatter of asymmetry increases toward the smallest amplitudes. Both scatter plots in Fig. 12 look qualitatively similar to the distributions observed by Sigwarth et al. (1999) in the quiet Sun in the same spectral line. In the observational data, a part of the scatter at small amplitudes is due to noise, however.

$2 \mathrm{D}$ radiation MHD simulations of solar photospheric magnetoconvection are also able to reproduce the observed values of the amplitude asymmetry. However, the area asymmetry in $2 \mathrm{D}$ simulations is always close to 0 (Steiner 1999; Sheminova 2003, 2004). It was concluded that the Stokes $V$ area asymmetry of spatially unresolved profiles is a result of a delicate balance between negative and positive contributions due to the structure of the magnetic and velocity fields in and around magnetic elements.

The present simulations allowed for the first time to obtain a significant non-zero area asymmetry. Although an exact match to the data was not yet achieved, the qualitative agreement is good. Therefore most of the essential ingredients must be present in the simulations to reproduce the observed asymmetry values. Thus we believe that the magnetic field and velocity gradients are in principle sufficient. The fact that $\delta A$ does not scale with $\langle B\rangle$ in the same way as observed may have different origins. E.g., the closed upper boundary affects the velocity structure to some extent. Subtle effects such as the possibility that the fraction of unipolar fields probably increases with $\langle B\rangle$ in the observations may also play a role. The magnetic Reynolds number of the simulation may also be an important factor, since in the simulation the thickness of the magnetic boundary layers, into which downflowing material at the edges of magnetic features can get entrained, is certainly larger than one expects for typical Reynolds numbers in the photosphere.

\section{Zero-crossing velocity}

There has been much debate about the presence or absence of net flows inside solar magnetic elements observed in solar plages, network and internetwork regions (see e.g. Solanki \& Stenflo 1986; Solanki \& Pahlke 1988; Grossmann-Doerth et al. 1996; Bellot Rubio et al. 1997; Frutiger \& Solanki 1998; Sigwarth et al. 1999; Sánchez Almeida \& Lites 2000). Usually, the Stokes $V$ zero-crossing wavelength is used to determine flow speeds within magnetic features, in particular the wavelength shift relative to the spatially averaged $I$ profile. The wavelength position of the Stokes $I$ profile averaged spatially and temporally, $\overline{I(\lambda)}$, contains a residual blueshift as a result of the spatially unresolved convective motions (Dravins et al. 1981). The average magnitude of the convective blueshift is about $200 \mathrm{~m} \mathrm{~s}^{-1}$, but the exact value depends on the excitation potential and depth of spectral line (Dravins et al. 1981; Allende Prieto \& García López 1998). If instead of averaging the intensity profile, one performs an average of its wavelength shifts $\lambda_{I}$, then the residual blueshift decreases as granulation becomes spatially resolved (see the Appendix in Keil et al. 1999). Thus, the line blueshift defined in this way will depend on the resolution of observations.

Consider first the wavelength position of the Stokes $I$ profile averaged over a snapshot $\overline{I(\lambda)}$. Such a profile is blueshifted. This blueshift is, on average, $120 \mathrm{~m} \mathrm{~s}^{-1}$ (Fe I $\left.6302 \AA\right)$ and $330 \mathrm{~m} \mathrm{~s}^{-1}$ (Fe I $15648 \AA$ ) in the snapshots with $\langle B\rangle$ between 10 and $80 \mathrm{G}$. The values for the IR lines are larger because they are formed deeper where the convective velocities are higher (see for example Asplund et al. 2000). The value of the blueshift for the Fe I $6302 \AA$ line is lower than obtained from observations (Dravins et al. 1981; Sigwarth et al. 1999). The blueshift 
of the $\overline{I(\lambda)}$ in the $\langle B\rangle=140 \mathrm{G}$ snapshot is larger for both lines and amounts to $500 \mathrm{~m} \mathrm{~s}^{-1}$ and $880 \mathrm{~m} \mathrm{~s}^{-1}$, in the visible and infrared, respectively. A possible reason for that is poor statistics. To derive statistically valid wavelength positions of the Stokes $I$ minimum and zero-crossing of Stokes $V$ the temporal averaging is necessary in addition to spatial averaging. The individual snapshots contain oscillations (see for example Asplund et al. 2000) and thus, the average over the snapshot $\lambda_{I}$ and $\lambda_{V}$ are expected to vary, depending on their (unknown) phase.

The average Stokes $I$ shift obtained by averaging shifts $\lambda_{I}$ instead of averaging the profiles themselves depends on the spatial resolution. At the original resolution, the average $\overline{\lambda_{I}}$ vary slightly around zero from one snapshot to another. At the reduced resolution the $\overline{\lambda_{I}}$ values are similar to those obtained by averaging the profiles (see values above).

The average value of $\lambda_{V}$ depends on the spatial resolution as well. We obtained $\overline{\lambda_{V}}$ by averaging the shifts from individual pixels and measured it with respect to the average $\bar{\lambda}_{I}$, both in the case of the original and reduced resolution. In the case of the original resolution, all regular-shaped profiles irrespective on their amplitudes were considered. The $\overline{\lambda_{V}}-\overline{\lambda_{I}}$ turned out to vary slightly around zero value for all the snapshots.

For reduced resolution, we measured zero-crossing wavelengths only for regular profiles with amplitude above $10^{-3}$. The value of $\overline{\lambda_{V}}-\overline{\lambda_{I}}$ increases to about $1 \mathrm{~km} \mathrm{~s}^{-1}$ (Fe I $15648 \AA$ ) and $850 \mathrm{~m} \mathrm{~s}^{-1}-1 \mathrm{~km} \mathrm{~s}^{-1}$ (Fe I $6302 \AA$ ) for $\langle B\rangle=10-80 \mathrm{G}$. In the $\langle B\rangle=140 \mathrm{G}$ case, the $\overline{\lambda_{V}}-\overline{\lambda_{I}}$ are the lowest for both lines and amount to $520 \mathrm{~m} \mathrm{~s}^{-1}$ (IR) and $340 \mathrm{~m} \mathrm{~s}^{-1}$ (visible).

The shift of the average $\overline{\lambda_{V}}$ position toward the red after reducing the resolution is easily understood. The main contribution comes from the intergranular lanes since the amplitudes of Stokes $V$ profiles are the strongest there. Thus, the resulted profiles will be contaminated by intergranular profiles and more redshifted.

The values of the zero-crossing velocity given in the literature are difficult to inter compare. This is due to the different wavelength offset of the measurements (average Stokes I position, absolute velocity calibration, etc.). In general, the values given for the enhanced magnetic field regions like plages are low and stay within the errors of the measurements (Solanki \& Stenflo 1986; Solanki \& Pahlke 1988; Martínez Pillet et al. 1997). In quiet network fields Stokes $V$ is shifted by $730 \mathrm{~m} \mathrm{~s}^{-1}$ (with respect to the laboratory wavelength of the Fe I $6302 \AA$ Sigwarth et al. 1999) and $970 \mathrm{~m} \mathrm{~s}^{-1}$ (with respect the the average Stokes $I$ position Grossmann-Doerth et al. 1996). In the internetwork, Khomenko et al. (2003) measured $\bar{\lambda}_{V}$ of the Fe I $15648 \AA$ of $550 \mathrm{~m} \mathrm{~s}^{-1}$ with respect to the blue-shifted Stokes $I$ profile and concluded that this value is compatible with the absence of a net velocity shift. Thus, the simulations give somewhat larger velocity shifts than are actually observed. A possible source of the discrepancy is a $1.5 \mathrm{D}$ spectral synthesis in the simulations. If a fully $3 \mathrm{D}$ radiative transfer is solved, horizontal flows are taken into account which may change the wavelength shifts of the resulting profiles. On the other hand, a significant part of the discrepancy can be due to excess difusivity in the models, which causes the material in the magnetic elements to be coupled to the downflows in the surrounding intergranular lanes.
What is the reason for the lower wavelength shift in the larger flux case of $\langle B\rangle=140 \mathrm{G}$ ? The distributions of the LOS velocities in the snapshots measured in all the points is very much similar for all the $\langle B\rangle$. Assume now, that we consider only the points with the amplitude above a certain threshold. Those will be the points that contribute most to the measured signal under the reduced resolution. In the case of the lowerflux snapshots, all these points are located in intergranules and, thus, the resulted signal is red-shifted. In the $140 \mathrm{G}$ case the overall signal is stronger and thus, granules contribute to the large-amplitude signals as well. This leads to the decrease of the average zero-crossing shifts for the larger-flux snapshots.

Another important reason for lower wavelength shifts in the large-flux snapshots is a significant reduction of the velocities in the interior of large-flux concentrations in intergranular lanes. These concentrations are stronger and the reduction is more significant in the large-flux snapshots than in the lowerflux ones (see lower panels in Fig. 1).

Finally, we note that the presence of a Stokes $V$ zerocrossing shift does not imply the presence of a net flow within the magnetic features in the resolution, since combinations of up- and downflows can produce shifted $V$ profiles even in the absence of a net mass flux (see, e.g., Grossmann-Doerth et al. 1991; Frutiger \& Solanki 2001).

\section{Comparison of IR and visible data}

Observations of the quiet Sun show a systematic difference in the properties of the magnetic field derived from visible and infrared spectral lines. The visible lines show larger field strengths and fluxes accompanied by lower filling factors (e.g. Grossmann-Doerth et al. 1996; Sánchez Almeida \& Lites 2000; Socas-Navarro \& Sánchez Almeida 2002; Sánchez Almeida et al. 2003b; Domínguez Cerdeña et al. 2003), compared to the IR (Khomenko et al. 2003). It was argued that the two sets of lines trace different parts of the magnetic field distribution. However, the results by different authors are difficult to compare since the observations were taken independently and at different times in the IR and the visible lines. Simultaneous observations have been made by Sánchez Almeida et al. (2003b) at two different telescopes (VTT and THEMIS) located at the Observatorio del Teide in Tenerife. The PCA analysis of the observed profiles showed that about a quarter of all significant profiles have different polarities in the IR and the visible spectral regions. The authors interpret this result as a confirmation that both spectral lines trace different magnetic structures in the same resolution element. However, since the polarization signals produced by the inter-network fields are very weak, the results of the observations are strongly influenced by the seeing conditions (compare, e.g. profile parameters before and after smearing in earlier sections). The seeing conditions at the sites of the two telescopes may vary significantly which is confirmed by the difference in the spatial resolution and granulation contrast obtained by Sánchez Almeida et al. (2003b) in the two spectral regions. The resolution of their IR observations is rather fair while the resolution in the visible is much worse. 
In the previous sections we have seen that the investigated simulations produce results that agree reasonably well with sensitive observations. Therefore we use the simulations to predict the results of simultaneous observations in the IR and visible lines. To this end, we perform a simultaneous PCA classification of the Fe I $15648 \AA$ and Fe I $6302 \AA$ Stokes $V$ profiles for three cases: (1) profiles with the original resolution; (2) profiles smeared with the visible and IR lines being treated identically; (3) smeared profiles with the visible line being smeared more strongly that the IR line. The second classification corresponds to the case of good seeing conditions in both spectral regions. In this case we use the parameters of the smoothing functions given in Sect. 4 for both spectral lines. In the last case, these parameters have been chosen such that profiles after the convolution have continuum contrast $\delta I_{\mathrm{c}}=1.7 \%$ and a resolution of $1^{\prime \prime}$ in the infrared and $\delta I_{\mathrm{c}}=1.9 \%$ and a resolution of 1".4 in the visible, values close to those of the observations by Sánchez Almeida et al. (2003b). The telescope diameter was taken to be $1 \mathrm{~m}$ (corresponding to the THEMIS telescope; for Fe I $6302 \AA$ ) and $70 \mathrm{~cm}$ (VTT; for Fe I $15648 \AA$ ). No noise was added to the profiles in order to separate the effects of noise from the effects of different seeing conditions.

Figure 13 illustrates the results of this test for the $\langle B\rangle=$ $140 \mathrm{G}$ snapshot. The other snapshots give similar results. The original profiles have similar shapes in the IR and in the visible at the same spatial points, with the asymmetries in the visible being stronger in a number of locations. The comparison of the profile polarity images reveals that they are exactly the same: the areas where the $V$ profiles show a particular polarity correspond to each other and the irregular profiles of both lines are located at the same pixels.

If the visible and the infrared profiles are smeared similarly, the asymmetries of the profiles in both spectral regions are still very similar (middle panel of Fig. 13). This is testified by the images of the polarity distribution, that correspond to each other almost one to one. We found no case in any of the snapshots where the visible and IR profiles both had 2 lobes and opposite polarities. The irregular profiles also appear at the same pixels. Only rarely, in about $1 \%$ of all the classified cases, does a normal profile in the infrared correspond to an irregular profile in the visible. The opposite case is even rarer.

This situation changes if the synthetic seeing conditions are significantly different for the IR and the visible. New classes of profiles appear (bottom panels of Fig. 13). In particular, there are classes of profiles with opposite polarities in the IR and the visible (an example is shown in the figure, where this is close to being the case), similar to ones obtained by Sánchez Almeida et al. (2003b). The number of the profiles belonging to such classes in the simulations makes $9 \%$ of all classified profiles. In addition, in $10 \%$ of the cases a 2-lobed profile of Fe I $15648 \AA$ corresponds to a 3 -lobed profile of Fe I $6302 \AA$, while in $5 \%$ of cases the reverse is true. Now the images of the polarity distribution are distinctly different, with large patches where either the polarity of the profiles is not the same (like in the upper right corner) or the irregular profiles of one spectral line correspond to normal profile of the other (bottom central part). The profiles of the classes like $\mathbf{c}$ and $\mathbf{d}$ shown in the bottom panels of Fig. 13 (different amounts of smearing) can form patches of $500-2000 \mathrm{~km}$ size.

In summary, differences in the seeing affecting visible and infrared observations can introduce spurious new classes of profiles. Therefore great care must be taken when analyzing observations from different sources or telescopes. If the seeing conditions are similar the Stokes $V$ asymmetries of the Fe I 15648 and Fe I 6302 lines are rather close to each other and they display the same polarity. Consequently, they trace the same magnetic structures in the resolution element.

\section{Discussion and conclusions}

We have used recent realistic 3D magnetoconvection simulations to study the polarization signals produced in the quiet Sun. These simulations aim at a realistic description of conditions around the solar surface. The particular set of simulations considered here take into account the mixture of polarities on a small patch of the solar surface. They allowed us to synthesize the Stokes spectra of some frequently used photospheric spectral lines. The amplitudes, asymmetries, shapes and zerocrossing shifts of the synthetic profiles were studied both for the original spatial resolution of the simulations and at a resolution typical of good spectropolarimetric observations. Different amounts of magnetic flux in the model box were also considered, although in a relatively limited range. The results were compared with observations in a statistical sense. A number of conclusions can be drawn from this study.

The comparison with observations confirms the simulations to be relatively realistic. The synthetic spectra have many properties in common with those observed in solar network and inter-network regions, in particular:

(i) The width and depth of the Stokes I (intensity) profile are reproduced reasonably well by the simulations.

(ii) The continuum images of simulation snapshots show the existence of bright points in intergranular lanes for average magnetic field strengths in the box as low as 20-30 G, which corresponds to an internetwork region.

(iii) The amplitude and area asymmetries of the Stokes $V$ profiles at $\sim 1^{\prime \prime}$ resolution are, on average, positive. The amplitude asymmetry $\delta a$ decreases with increasing flux in the simulation box. Both $\delta A$ and $\delta a$ for the $\langle B\rangle=140 \mathrm{G}$ snapshot are close to the values observed in the solar network regions.

(iv) The average area asymmetry of the Stokes $V$ profiles at $\sim 1^{\prime \prime}$ resolution is positive in granules and negative or close to zero in intergranules.

(v) The classes of the $15648 \AA$ Stokes $V$ profiles under reduced resolution are similar to those actually observed. The profiles belonging to the same classes are clustered together and form patches on the surface close to the spatial resolution in size.

(vi) Under reduced resolution, the irregular-shaped Stokes $V$ profiles appear between areas of opposite polarity. The abundance of the irregular-shaped $V$ profiles with amplitudes above $10^{-3}$ increases with decreasing average magnetic field strength from $16-17 \%$ for $\langle B\rangle=140 \mathrm{G}$ to about 

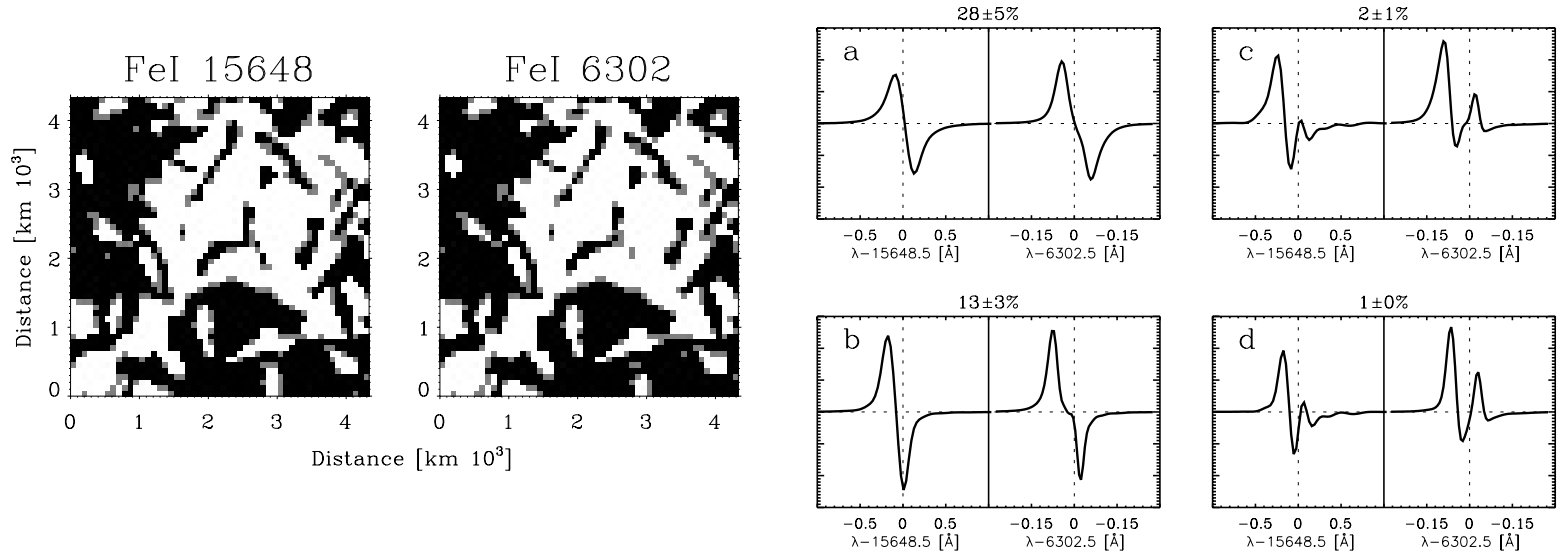

SAME AMOUNT OF SMEARING

FeI 15648

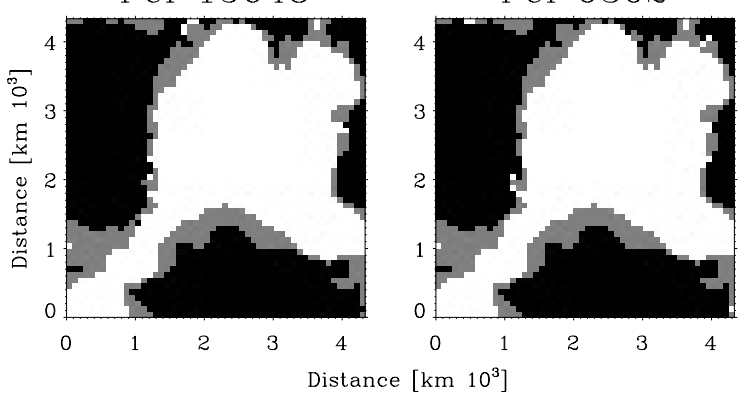

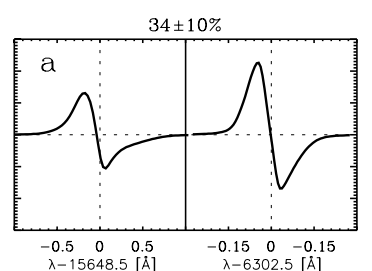

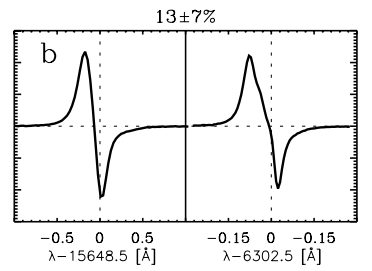

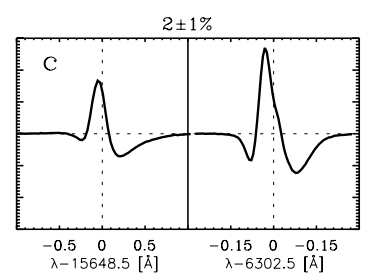

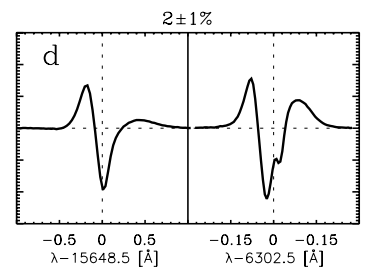

DIFFERENT AMOUNT OF SMEARING

FeI 15648

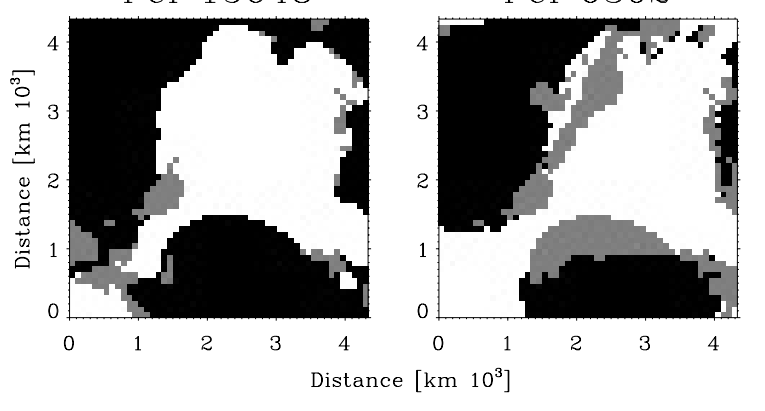

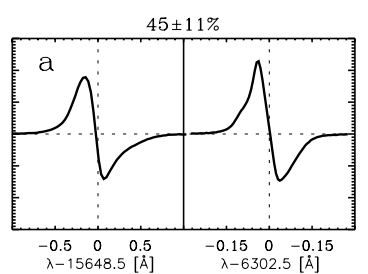

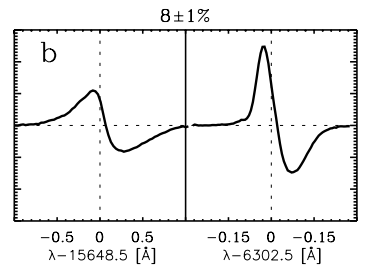

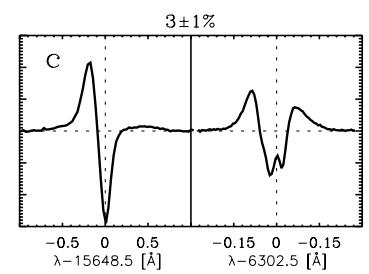

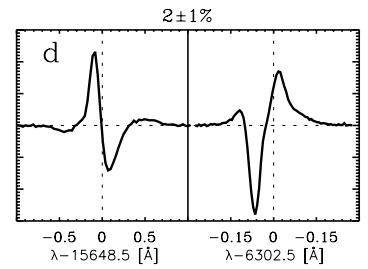

Fig. 13. Simultaneous classification of the Fe I $15648 \AA$ and Fe I $6302 \AA$ Stokes $V$ profiles in the $\langle B\rangle=140 \mathrm{G}$ snapshot. Upper panel: original resolution; middle panel: same amount of smearing at both wavelengths; bottom panel: different amounts of smearing (see text for details). The images show the spatial polarity distribution for both spectral lines. Black and white colors mark the locations of the positive and negative polarity profiles, while grey marks the location of the irregular profiles. To make the comparison easier, only about $2 / 3$ of the original snapshot is shown (upper right part). The examples of the class profiles corresponding to each classification are given on the right hand side of the figure together with the fraction of the profiles belonging to each class.

$30 \%$ for $\langle B\rangle=20 \mathrm{G}$. These values are in good agreement with observations.

Our analysis also revealed some features where there is still a discrepancy between the simulations and observations. Observed features that are not well reproduced by the synthetic spectra and therefore suggest the need for further improvement of the simulations are:

(i) At $\sim 1^{\prime \prime}$ resolution, the amplitude asymmetry is too high while the area asymmetry is too low for the low-flux 
snapshots of 10-30 G, as compared to the observations of the magnetic field in the solar inter-network region.

(ii) The zero-crossing shifts in the simulations are higher than observed in the quiet Sun.

In addition, the present investigation has led to new insights into solar magnetic fields partly because the simulations allow profiles at a much higher spatial resolution to be studied than possible with current observational instruments. The simulations also provide guidance for the improved interpretation of observations:

(i) The profiles with original resolution are already very asymmetric. The positive and negative asymmetries are not in balance and the average amplitude and area asymmetry is in general positive.

(ii) The real abundance of the irregular-shaped Stokes $V$ profiles at $\sim 1^{\prime \prime}$ resolution is 1.5 times larger than is revealed by the observations. In current observations most of these profiles remain below the noise level. Interestingly, the abundance of the irregular profiles revealed by real noisy observations is close to their "true" abundance existing at the original resolution of the simulations.

(iii) The presence of irregular profiles under reduced resolution reflects more the horizontal than the vertical structuring of the magnetic fields since the spatial location of such profiles depends strongly on the spatial resolution. This conclusion is independent of whether or not noise is added to the synthetic profiles.

(iv) The shapes of the Fe I $15648 \AA$ and $6302 \AA$ Stokes $V$ profiles observed simultaneously under similar seeing conditions are very close to each other. This suggests that infrared and visible spectral lines trace the same magnetic structures in the resolution element. If the seeing conditions under which the visible and IR data were recorded differ significantly, new classes of profiles appear. In particular, the polarization signals can have opposite signs in the infrared and in the visible. These profiles can occupy areas of 500-2000 km size. This results underlines the importance of data obtained under nearly identical seeing conditions in the two spectral bands.

(v) The average $\lambda_{V}$ position shows no net redshift for the original resolution profiles. The redshift appears after smearing due to selection effects introduced by considering only the large-amplitude profiles located in intergranules. This result, however, must be considered with some caution, since we do not average over a sufficiently large sample of snapshots to remove the effects of oscillations.

(vi) The amplitudes of the $15648 \AA$ Stokes $V$ signals observed in the inter-network are consistent with the amplitudes in the simulation snapshot having an average magnetic field strength of $\langle B\rangle=20 \mathrm{G}$. In observations with a spatial resolution close to $1^{\prime \prime}$ up to $2 / 3$ of the original flux can remain undetected if one uses amplitudes as a measure of the magnetic flux, even if the noise level of the observations is infinitely low (e.g., Khomenko et al. 2005).

The last conclusion seems to be the most important and needs a special discussion. There has been much debate about the amount of the magnetic flux contained in the quiet Sun. The value of $20 \mathrm{G}$ "real" spatially averaged magnetic field strength implies that values of $\langle B\rangle=7-8 \mathrm{G}$ would be detected at $\sim 1^{\prime \prime}$ resolution observations, since only $1 / 3$ of the magnetic flux is expected to be visible at this resolution. Polarimetric observations of the Zeeman effect reported in the literature give values of the longitudinal magnetic flux component $\left\langle\left|B_{z}\right|\right\rangle$ rather that the average field strength $\langle B\rangle$. The former is lower and decreases from 15 to 11 from $\log \tau=0$ to -1 in the simulation snapshot with $\langle B\rangle=20 \mathrm{G}$. It means that the longitudinal flux detected at $\sim 1^{\prime \prime}$ resolution in the observations with infinite polarimetric sensitivity should be $\left\langle\left|B_{z}\right|\right\rangle=4-5 \mathrm{G}$. Since the observations reported in the literature have a significant level of noise, the signals are measured only in some $30-60 \%$ of the field of view. Consequently, only the highest-amplitude signals are detected and the magnetic flux averaged over these points is larger than the average over the whole area. Many inter-network observations provide values of 6-11 G. For example, Advanced Stokes Polarimeter data give values of about 8-9 G (Lites 2002; Lites \& Socas-Navarro 2004). The latter authors claim that the measured flux does not change even if the resolution is improved to $0{ }^{\prime} 6$. The Tenerife Infrared Polarimeter observations at $1^{\prime \prime}$ resolution give $8 \mathrm{G}$ (Khomenko et al. 2003). In the simultaneous observations in the visible and infrared reported by Sánchez Almeida et al. (2003b) the flux in both spectral ranges is not the same and amounts to $11 \mathrm{G}$ (Fe I $6302 \AA$ ) and 6 G (Fe I $15648 \AA$ ). The flux obtained in the visible by these authors is larger than in the similar ASP data even though the resolution of the former observations was significantly lower (about 1 .' 4 ). The results of the simulations are in apparent contradiction with the high-resolution ( $\left.0 .{ }^{\prime} 5\right)$ observations by Domínguez Cerdeña et al. (2003) who measured fluxes between 17 and $21 \mathrm{G}$ from the $60 \%$ of the area covered by the signal above noise in their data.

All the above results are based on measurements of the Zeeman effect. Using the Hanle effect and employing a 3D hydrodynamical simulations Trujillo Bueno et al. (2004) determined an averaged unsigned magnetic field strength of up to $60 \mathrm{G}$ in the inter-network (see also Bommier et al. 2005; Manso Sainz et al. 2005). Since the field is assumed to be turbulent, all the directions are equally probable and the longitudinal flux of such a field should measure about $60 / \sqrt{3} \approx 35 \mathrm{G}$. The apparent contradictions between these measurements and our estimations may not be real since both techniques are affected by simplifications. For example, the amplitudes of Zeeman polarization signal in the investigated Fe I lines may change if spectral synthesis is done in 3D. However, possibly, the analysis done by Trujillo Bueno et al. (2004) is pointing to the need for more turbulent field in the simulations.

Finally, the overall good agreement of simulations with the different types of observations, which include not just the spectropolarimetric data considered here, but also $G$-band observations (Schüssler et al. 2003; Shelyag et al. 2004; Keller et al. 2004), confirms that most of the essential physical ingredients are already present in the models. Some discrepancies remain, however, which point to the need to improve the simulations even further. One obvious improvement concerns a more realistic upper boundary, which might improve the temperature 
distribution in the upper layers. A higher numerical spatial resolution should also affect the results of the simulations. Higher grid resolution will lead to thinner diffusive boundary layers at the edges of strong flux concentrations. Since at these boundary layers the strong fields and strong downflow velocities coincide, grid resolution effects should affect the area and amplitude asymmetries as well as the Stokes $V$ zero-crossing shift and, probably, decrease the discrepancies. Finally it cannot be ruled out that NLTE 3D computations will be required to remove all the discrepancies. The simulations can be used for further studies of the magnetic field properties in the quiet solar regions. Among the questions that can be addressed by the simulations are the origin of the amplitude and area asymmetries of Stokes profiles; the observed discrepancies in the magnetic flux and distribution of the magnetic field strength recovered from the different spectral lines under limited spatial resolution and the reliability of widely used diagnostics and line profile inversions.

Acknowledgements. We are grateful to M. Schüssler, N. Shchukina, V. Sheminova, O. Steiner and M. Collados for discussions and J. M. Borrero for help with atomic parameters. This work was partially supported by INTAS grant 00-00084 and by the Spanish Ministerio de Educación y Ciencia through project AYA2004-05792.

\section{References}

Allende Prieto, C., \& García López, R. J. 1998, A\&AS, 129, 41

Anstee, S. D., \& O'Mara, B. J. 1995, MNRAS, 276, 859

Asplund, M., Nordlund, Å., Trampedach, R., Allende Prieto, C., \& Stein, R. F. 2000, A\&A, 359, 729

Barklem, P. S., Anstee, S. D., \& O’Mara, B. J. 1998, Proc. Astron. Soc. Austr., 15, 336

Barklem, P. S., Piskunov, N., \& O’Mara, B. J. 2000, A\&AS, 142, 467

Bellot Rubio, L. R., Ruiz Cobo, B., \& Collados, M. 1997, ApJ, 478, L45

Bommier, V., Derouich, M., Landi Degl'Innochenti, E., Molodij, G., \& Sahal-Bréchot, S. 2005, A\&A, 432, 295

Cattaneo, F. 1999, ApJ, 515, L39

Delbouille, L., Neven, L., \& Roland, G. 1973, Photometric atlas of the solar spectrum from $\lambda 3000$ to $\lambda 10000$, Institut d'Astrophysique de l'Université de Liège, Liège, Belgium

Domínguez Cerdeña, I., Sánchez Almeida, J., \& Kneer, F. 2003, A\&A, 407, 741

Dravins, D., Lindegren, L., \& Nordlund, A. 1981, A\&A, 96, 345

Emonet, T., \& Cattaneo, F. 2001, ApJ, 560, L197

Frutiger, C., \& Solanki, S. K. 1998, A\&A, 336, L65

Frutiger, C., \& Solanki, S. K. 2001, A\&A, 369, 646

Frutiger, C. C., Solanki, S. K., Fligge, M., \& Bruls, J. H. M. J. 2000, A\&A, 358, 1109

Gadun, A. S., Solanki, S. K., Sheminova, V. A., \& Ploner, S. R. O. 2001, Sol. Phys., 203, 1

Grossmann-Doerth, U., Schüssler, M., \& Solanki, S. K. 1988, Sol. Phys., 206, L37

Grossmann-Doerth, U., Schüssler, M., \& Solanki, S. K. 1989, A\&A, 221,338

Grossmann-Doerth, U., Schüssler, M., \& Solanki, S. K. 1991, A\&A, 249,239

Grossmann-Doerth, U., Keller, C. U., \& Schüssler, M. 1996, A\&A, 315,610

Grossmann-Doerth, U., Schüssler, M., \& Steiner, O. 1998, A\&A, 337, 928
Grossmann-Doerth, U., Schüssler, M., Sigwarth, M., \& Steiner, O. 2000, A\&A, 357, 351

Holweger, H., \& Müller, E. 1974, Sol. Phys., 39, 19

Illing, R. M. E., Landman, D. A., \& Mickey, D. L. 1975, A\&A, 41, 183

Keil, S. L., Balasubramaniam, K. S., Smaldone, L. A., \& Reger, D. 1999, ApJ, 510, 422

Keller, C., Schüssler, M., Vögler, A., \& Zakharov, V. 2004, ApJ, 607, L59

Keller, C. U., Deubner, F.-L., Egger, U., Fleck, B., \& Povel, H. P. 1994, A\&A, 286, 626

Khomenko, E. V., Martínez González, M. J., Collados, M., et al. 2005, A\&A, accepted

Khomenko, E. V., Collados, M., Solanki, S. K., Lagg, A., \& Trujillo Bueno, J. 2003, A\&A, 408, 1115

Kupka, F., Piskunov, N. E., Ryabchikova, T. A., Stempels, H. C., \& Weiss, W. W. 1999, A\&AS, 138, 119

Kurucz, R. 1994, Atomic Data for Fe and Ni. Kurucz CD-ROM No. 22, Cambridge, Mass.: Smithsonian Astrophysical Observatory Lin, H. 1995, ApJ, 446, 421

Lites, B., \& Socas-Navarro, H. 2004, ApJ, 613, 600

Lites, B. W. 2002, ApJ, 573, 431

Manso Sainz, R., Landi Degl'Innocenti, E., \& Trujillo Bueno, J. 2005, ApJ, in press

Martínez Pillet, V., Lites, B. W., \& Skumanich, A. 1997, ApJ, 474, 810

Martínez Pillet, V., Collados, M., Sánchez Almeida, J., et al. 1999, in High resolution solar physics: theory, observations and techniques, ed. T. R. Rimmele, K. S. Balasubramaniam, \& R. R. Radick, ASP Conf. Ser., 183, 264

Nave, G., Johansson, S., Learner, R. C. M., Thorne, A. P., \& Brault, J. W. 1994, ApJS, 94, 221

Nordlund, Å. 1984, in Small-Scale Dynamical Processes in Quiet Stellar Atmospheres, Sunspot: Sacramento Peak Obs., ed. S. L. Keil, 174

Piskunov, N. E., Kupka, F., Ryabchikova, T. A., Weiss, W. W., \& Jeffery, C. S. 1995, A\&AS, 112, 525

Ploner, S. R. O., Schüssler, M., Solanki, S. K., \& Gadun, A. 2001, in Advanced solar polarimetry: Theory, observation and instrumentetion, ed. M. Sigwarth, ASP Conf. Ser., 236, 371

Rabin, D. 1992, ApJ, 391, 832

Rees, D. E., Durrant, D. J., \& Murphy, G. A. 1989, ApJ, 339, 1093

Rees, D. E., López Ariste, A., Thatcher, J., \& Semel, M. 2000, A\&A, 355,759

Rüedi, I., Solanki, S. K., Livingston, W., \& Stenflo, J. O. 1992, A\&A, 263,323

Ryabchikova, T. A., Piskunov, N. E., Stempels, H. C., Kupka, F., \& Weiss, W. W. 1999, in Proc. of the 6th International Colloquium on Atomic Spectra and Oscillator Strengths, Physica Scripta, Victoria BC, Canada, T83, 162

Sánchez Almeida, J., Collados, M., \& del Toro Iniesta, J. C. 1989, A\&A, 222, 311

Sánchez Almeida, J., \& Lites, B. W. 1992, ApJ, 398, 359

Sánchez Almeida, J., Landi degl'Innocenti, E., Martínez Pillet, V., \& Lites, B. W. 1996, ApJ, 466, 537

Sánchez Almeida, J., \& Lites, B. W. 2000, ApJ, 532, 1215

Sánchez Almeida, J., Emonet, T., \& Cattaneo, F. 2003a, ApJ, 585, 536

Sánchez Almeida, J., Domínguez Cerdeña, I., \& Kneer, F. 2003b, ApJ, 597, L177

Sánchez Almeida, J., Márquez, I. M., Bonet, J. A., Domínguez Cerdeña, I., \& Muller, R. 2004, ApJ, 609, L91

Schmidt, W., Kentischer, T. J., \& Lites, B. W. 2000, POLIS: Instrument Description (HAO-KIS) 
Schüssler, M. 2001, in Advanced solar polarimetry: Theory, observation and instrumentetion, ed. M. Sigwarth, ASP Conf. Ser., 236, 343

Schüssler, M. 2003, in Third International Workshop on Solar Polarization, ed. J. Trujillo Bueno, \& J. Sánchez Almeida, ASP Conf. Ser., 307, 601

Schüssler, M., Shelyag, S., Berdyugina, S., Vögler, A., \& Solanki, S. K. 2003, ApJ, 597, L173

Shchukina, N. G., \& Trujillo Bueno, J. 2001, ApJ, 550, 970

Shelyag, S. 2004, Spectro-polarimetric diagnostics of magnetoconvection simulations of the solar photosphere, Ph.D. Thesis, Univ. Göttingen

Shelyag, S., Schüssler, M., Solanki, S. K., Berdyugina, S. V., \& Vögler, A. 2004, A\&A, 427, 335

Shelyag, S., et al. 2005, A\&A, in preparation

Sheminova, V. A. 2003, Kinematika Fiz. Nebesn. Tel, 19 N 2, 107

Sheminova, V. A. 2004, Kinematika Fiz. Nebesn. Tel, 20 N 2, 3

Sigwarth, M. 2001, ApJ, 563, 1031

Sigwarth, M., Balasubramaniam, K. S., Knölker, M., \& Schmidt, W. 1999, A\&A, 349, 941

Socas-Navarro, H., \& Sánchez Almeida, J. 2002, ApJ, 565, 1323

Socas-Navarro, H., Martínez Pillet, V., \& Lites, B. 2004, ApJ, 611, 1139

Solanki, S. K. 1987, The Photospheric Layers of Solar Magnetic Fluxtubes, Ph.D. Thesis, ETH, Zurich
Solanki, S. K. 1989, A\&A, 224, 225

Solanki, S. K. 1993, Space Sci. Rev., 63, 1

Solanki, S. K., \& Montavon, C. A. P. 1993, A\&A, 275, 283

Solanki, S. K., \& Pahlke, K. D. 1988, A\&A, 201, 143

Solanki, S. K., Rüedi, I., \& Livingston, W. 1992, A\&A, 263, 312

Solanki, S. K., \& Stenflo, J. O. 1986, A\&A, 170, 120

Spruit, H. C. 1974, Sol. Phys., 34, 277

Steiner, O. 1999, in Advances in Solar Physics Euroconference: Magnetic Fields and Oscillations, ed. B. Schmieder, A. Hofmann, ASP Conf. Ser., 184, 38

Steiner, O. 2000, Sol. Phys., 196, 245

Steiner, O., Grossmann-Doerth, U., Knölker, M., \& Schüssler, M. 1998, ApJ, 495, 468

Stenflo, J. O. 1973, Sol. Phys., 32, 41

Trujillo Bueno, J., Shchukina, N. G., \& Asensio Ramos, A. 2004, Nature, 430, 326

Vögler, A. 2003, Three-dimentional simulations of magnetoconvection in the solar photosphere, Ph.D. Thesis, Univ. Göttingen

Vögler, A., \& Schüssler, M. 2003, Astron. Nachr., 324, 399

Vögler, A., Shelyag, S., Schüssler, M., et al. 2003, in Modelling of Stellar Atmospheres, ed. N. E. Piskunov, N. W. Weiss, \& D. E. Gray, ASP Conf. Ser., 210, 157

Vögler, A., Shelyag, S., Schüssler, M., et al. 2005, A\&A, 429, 335 